\title{
Pandora: nucleotide-resolution bacterial pan-genomics with reference graphs
}

Rachel M. Colquhoun ${ }^{1,2,3}$, Michael B. Hall' ${ }^{1}$ Leandro Lima', Leah W. Roberts ${ }^{1}$, Kerri M. Malone ${ }^{1}$, Martin Hunt ${ }^{1,4}$, Brice Letcher ${ }^{1}$, Jane Hawkey ${ }^{5}$, Sophie George ${ }^{4}$, Louise Pankhurst ${ }^{4,6}$ and Zamin Iqbal ${ }^{1 *}$ (i)

* Correspondence: zi@ebi.ac.uk ${ }^{1}$ European Bioinformatics Institute, Hinxton, Cambridge CB10 1SD, UK Full list of author information is available at the end of the article

\begin{abstract}
We present pandora, a novel pan-genome graph structure and algorithms for identifying variants across the full bacterial pan-genome. As much bacterial adaptability hinges on the accessory genome, methods which analyze SNPs in just the core genome have unsatisfactory limitations. Pandora approximates a sequenced genome as a recombinant of references, detects novel variation and pan-genotypes multiple samples. Using a reference graph of 578 Escherichia coli genomes, we compare 20 diverse isolates. Pandora recovers more rare SNPs than single-referencebased tools, is significantly better than picking the closest RefSeq reference, and provides a stable framework for analyzing diverse samples without reference bias.
\end{abstract}

Keywords: Pan-genome, Genome graph, Accessory genome, Nanopore

\section{Background}

Bacterial genomes evolve by multiple mechanisms including mutation during replication, allelic and non-allelic homologous recombination. These processes result in a population of genomes that are mosaics of each other. Given multiple contemporary genomes, the segregating variation between them allows inferences to be made about their evolutionary history. These analyses are central to the study of bacterial genomics and evolution [1-4] with different questions requiring focus on separate aspects of the mosaic: fine-scale (mutations) or coarse (gene presence, synteny). In this paper, we provide a new and accessible conceptual model that combines both fine and coarse bacterial variation. Using this new understanding to better represent variation, we can access previously hidden single-nucleotide polymorphisms (SNPs), insertions and deletions (indels). This can be used to add resolution to phylogenetic analyses of diverse cohorts, to investigate selection and adaptation in the accessory genome, and to aid bacterial genome-wide association studies (GWAS).

In the standard approach to analyzing genetic variation, a single genome is treated as a reference and all other genomes are interpreted as differences from it. This approach is problematic in bacteria, because while genes cover $85-90 \%$ of bacterial genomes [5], the full set of genes present in a bacterial species-the pan-genome-is in general

(c) The Author(s). 2021 Open Access This article is licensed under a Creative Commons Attribution 4.0 International License, which permits use, sharing, adaptation, distribution and reproduction in any medium or format, as long as you give appropriate credit to the original author(s) and the source, provide a link to the Creative Commons licence, and indicate if changes were made. The images or other third party material in this article are included in the article's Creative Commons licence, unless indicated otherwise in a credit line to the material. If material is not included in the article's Creative Commons licence and your intended use is not permitted by statutory regulation or exceeds the permitted use, you will need to obtain permission directly from the copyright holder. To view a copy of this licence, visit http://creativecommons.org/licenses/by/4.0/. The Creative Commons Public Domain Dedication waiver (http://creativecommons.org/publicdomain/zero/1.0/) applies to the data made available in this article, unless otherwise stated in a credit line to the data. 
much larger than the number found in any single genome. Further, the frequency distribution of genes has a characteristic asymmetric U-shaped curve [6-10], as shown in Fig. 1A. As a result, a single-reference genome will inevitably lack many of the genes in the pan-genome and completely miss genetic variation therein (Fig. 1B). We call this hard reference bias, to distinguish from the more common concern, that increased divergence of a reference from the genome under study leads to read-mapping problems, which we term soft reference bias. The standard workaround for these issues in bacterial genomics is to restrict analysis either to very similar genomes using a closely related reference (e.g., in an outbreak) or to analyze SNPs only in the core genome (present in most samples) and outside the core to simply study presence/absence of genes [11].

In this study, we address the variation deficit caused by a single-reference approach. Given Illumina or Nanopore sequence data from potentially divergent isolates of a bacterial species, we attempt to detect all of the variants between them. Our approach is to decompose the pan-genome into atomic units (loci) which tend to be preserved over evolutionary timescales. Our loci are genes and intergenic regions in this study, but the method is agnostic to such classifications, and one could add any other grouping wanted (e.g., operons or mobile genetic elements). Instead of using a single genome as a reference, we collect a panel of representative reference genomes and use them to construct a set of reference graphs, one for each locus. Reads are mapped to this set of graphs and from this we are able to discover and genotype variation. By letting go of prior information on locus ordering in the reference panel, we are able to recognize and genotype variation in a locus regardless of its wider context. Since Nanopore reads are typically long enough to encompass multiple loci, it is possible to subsequently infer the order of loci-although that is outside the scope of this study.

The use of graphs as a generalization of a linear reference is an active and maturing field [12-19]. Much recent graph genome work has gone into showing that genome

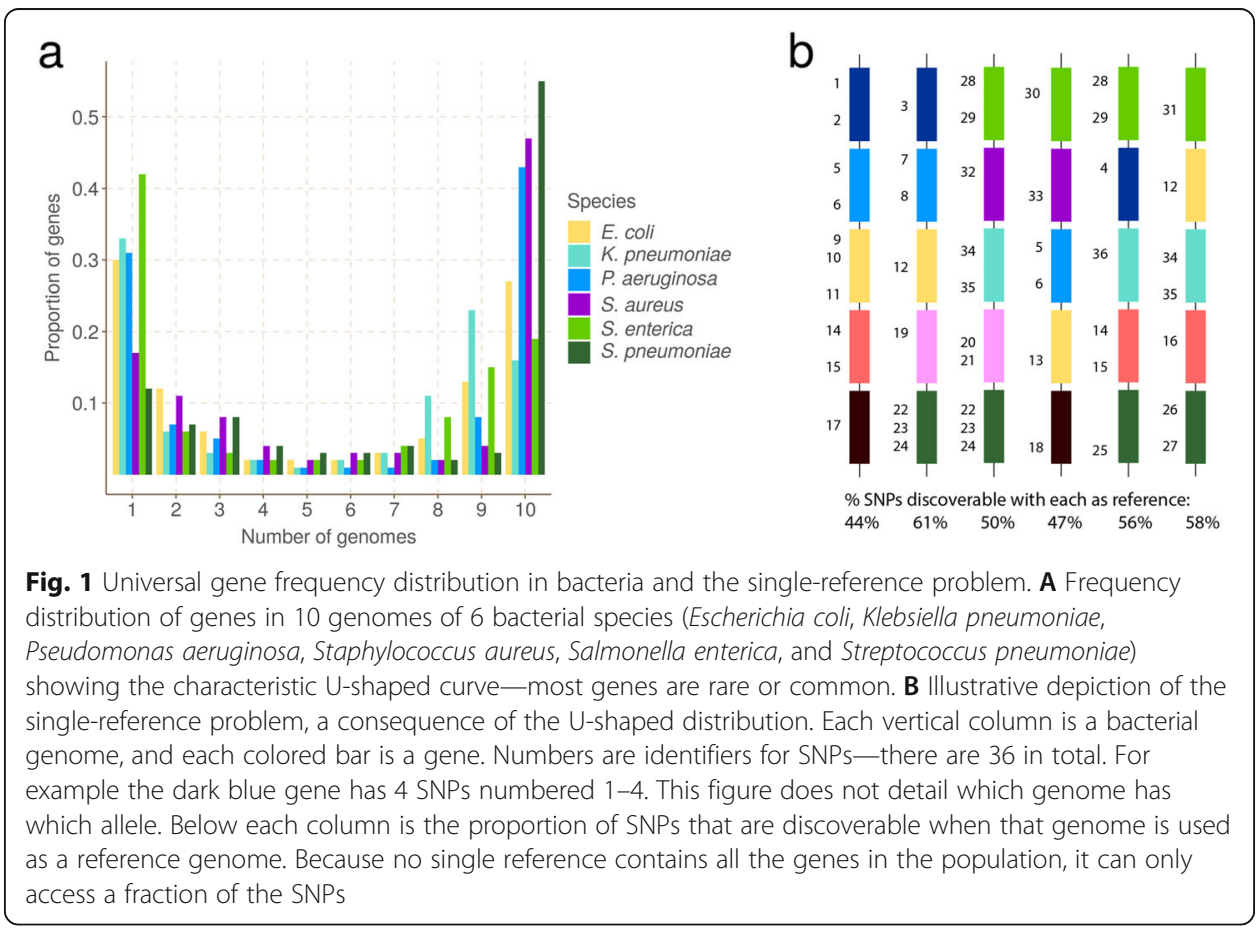


graphs reduce the impact of soft reference bias on mapping [12, 14, 15], and on generalizing alignment to graphs $[16,20]$. These methods have almost universally been designed for the human pan-genome, where hard reference bias is a comparatively minor issue compared with bacteria (two human genomes are over 99\% alignable, whereas two bacteria of the same species might be $50 \%$ alignable). In particular, all current graph methods (e.g., vg [12], Giraffe [21], GraphTyper [14, 15], paragraph [22], BayesTyper [23]) require a reference genome to be provided in advance to output genetic variants in the standard Variant Call Format (VCF) [24] - thus immediately inheriting a hard bias when applied to bacteria (see Fig. 1B). Thus, there has not yet been any study (to our knowledge) addressing SNP analysis across a diverse cohort, including more variants that can fit on any single reference.

We have made a number of technical innovations. First, we use a recursive clustering algorithm that converts a multiple sequence alignment (MSA) of a locus into a graph. This avoids the complexity "blowups" that plague graph genome construction from unphased VCF files [14,15]. Second, we introduce a graph representation of genetic variation based on $(w, k)$-minimizers [25]. Third, using this representation, we avoid unnecessary full alignment to the graph and instead use quasi-mapping to genotype on the graph. Fourth, we use local assembly to discover variation missing from the reference graph. Fifth, we infer a canonical dataset-dependent reference genome designed to maximize clarity of description of variants (the value of this will be made clear in the main text).

We describe these below and evaluate our implementation, pandora, on a diverse set of E. coli genomes with both Illumina and Nanopore data. We show that, compared with reference-based approaches, pandora recovers a significant proportion of the missing variation in rare loci, performs much more stably across a diverse dataset, successfully infers a better reference genome for VCF output, and outperforms current tools for Nanopore data.

\section{Results}

Pan-genome graph representation

We set out to define a generalized reference structure which allows detection of SNPs and other variants across the whole pan-genome, without attempting to record longrange structure or coordinates. We define a Pan-genome Reference Graph (PanRG) as an unordered collection of sequence graphs, termed local graphs, each of which represents a locus, such as a gene or intergenic region. Each local graph is constructed from a MSA of known alleles of this locus, using a recursive cluster-and-collapse (RCC) algorithm (Additional file 1: Supplementary Animation 1: recursive clustering construction). The output is guaranteed to be a directed acyclic sequence graph allowing hierarchical nesting of genetic variation while meeting a "balanced parentheses" criterion (see Fig. 2B and "Methods"). Each path through the graph from source to sink represents a possible recombinant sequence for the locus. The disjoint nature of this pan-genome reference allows loci such as genes to be compared regardless of their wider genomic context. We implement this construction algorithm in the make_prg tool which outputs the graph as a file (see Fig. 2A-C, "Methods"). We also implement a PanRG update algorithm in make prg which allows rapid augmentation of a pre-built PanRG with new alleles (see Fig. 2D, 


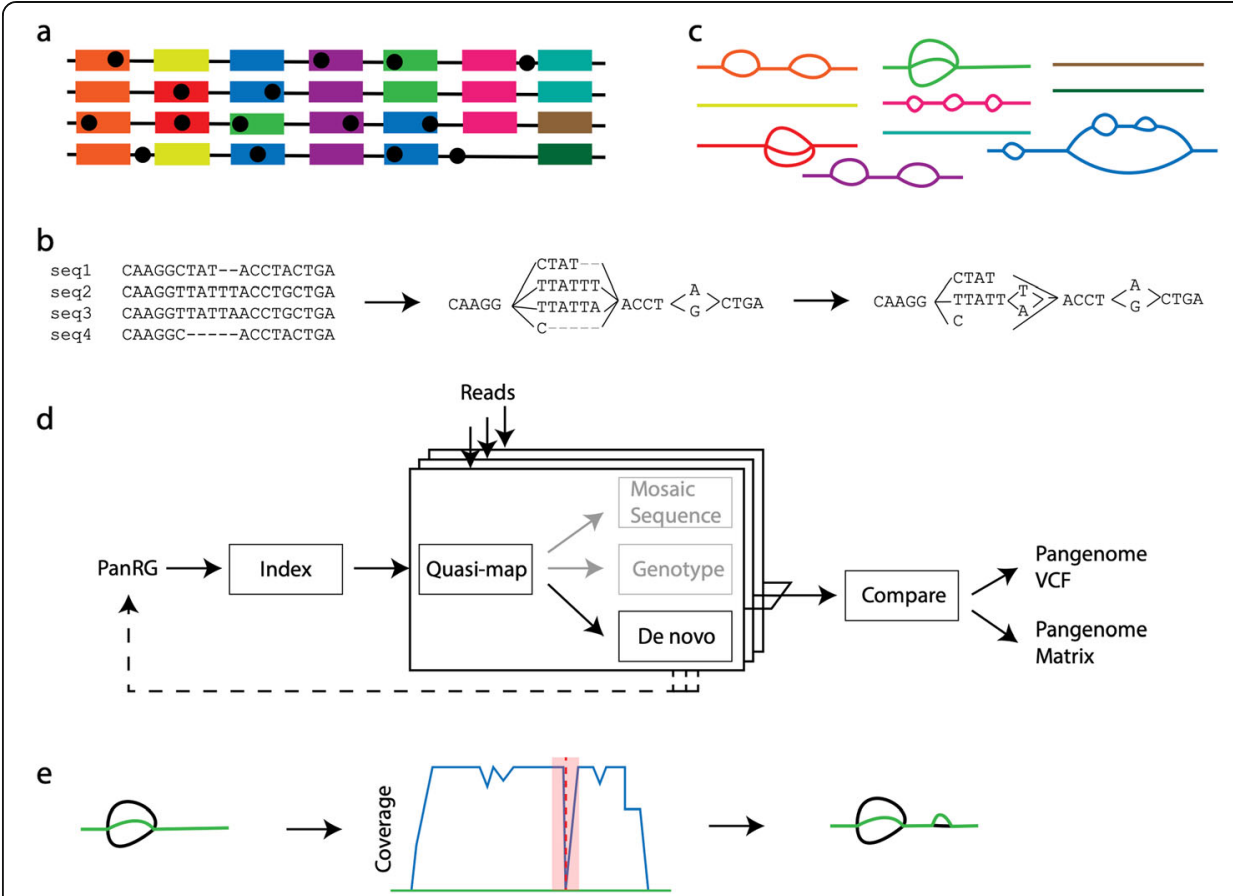

Fig. 2 The pandora workflow. A Reference panel of genomes; color signifies locus (gene or intergenic region) identifier, and blobs are SNPs. B The multiple sequence alignment (MSA) for each locus is converted into a directed acyclic graph (termed local graph). C Local graphs constructed from the loci in the reference panel. D Workflow: the collection of local graphs, termed the PanRG, is indexed. Reads from each sample under study are independently quasi-mapped to the graph, and a determination is made as to which loci are present in each sample. In this process, for each locus, a mosaic approximation of the sequence for that sample is inferred, and variants are genotyped. E Regions of low coverage are detected, and local de novo assembly is used to generate candidate novel alleles missing from the graph. Returning to $\mathbf{D}$, the dotted line shows all the candidate alleles from all samples are then gathered and added to the PanRG. Then, reads are quasi-mapped one more time, to the augmented PanRG, generating new mosaic approximations for all samples and storing coverages across the graphs; no de novo assembly is done this time. A pan-genome matrix showing which input loci are present in each sample is created. Finally, all samples are compared, and a VCF file is produced, with a per-locus reference that is inferred by pandora

"Methods"). Subsequent operations, based on this PanRG, are implemented in the software package pandora. The overall workflow is shown in Fig. 2.

To index a PanRG, we generalize a type of sparse marker $k$-mer $((w, k)$-minimizer, also referred to as a minimizing $k$-mer), previously defined for strings, to directed acyclic graphs (see "Methods"). Informally, each minimizer is the smallest $k$-mer in a window of $w$ consecutive $k$-mers. This has become a popular method for rapidly indexing and comparing genomes and long reads (e.g., MASH [26], minimap [27, 28]). It has the advantage that the number of indexing $k$-mers scales with the length of the sequence so that different length local graphs are each well represented in the index. In addition, it enables shorter indexing $k$-mers to be used, which improves mapping with noisy reads.

Each local graph is sketched with minimizing $k$-mers, and these are then used to construct a new graph (the $k$-mer graph) for each local graph from the PanRG. Each minimizing $k$-mer is a node, and edges are added between two nodes if they are adjacent minimizers on a path through the original local graph. This $k$-mer graph is isomorphic to the original if $w \leq k$ (and outside the first and last $w+k-1$ bases); all subsequent operations are performed on this graph, which, to avoid unnecessary new terminology, we also call the local graph. 
A global index maps each minimizing $k$-mer to a list of all local graphs containing that $k$-mer and the positions therein. Long or short reads are approximately mapped (quasi-mapped) to the PanRG by determining the minimizing $k$-mers in each read. Any of these read quasi-mappings found in a local graph are called hits, and any local graph with sufficient clustered hits on a read is considered present in the sample.

\section{Initial sequence approximation as a mosaic of references}

For each locus identified as present in a sample, we initially approximate the sample's sequence as a path through the local graph. The result is a mosaic of sequences from the reference panel. This path is chosen to have maximal support by reads, using a dynamic programming algorithm on the graph induced by its $(w, k)$-minimizers (details in "Methods"). The result of this process serves as our initial approximation to the genome under analysis.

\section{Improved sequence approximation: modify mosaic by local assembly}

At this point, we have quasi-mapped reads, and approximated the genome by finding the closest mosaic in the graph; however, we expect the genome under study to contain variants that are not present in the PanRG. Therefore, to allow discovery of novel SNPs and small indels that are not in the graph, for each sample and locus, we identify regions of the inferred mosaic sequence where there is a drop in read coverage (as shown in Fig. 2E). Slices of overlapping reads are extracted, and a form of de novo assembly is performed using a de Bruijn graph. Instead of trying to find a single correct path, the de Bruijn graph is traversed (see "Methods" for details) to obtain all feasible candidate novel alleles for the sample. These alleles are then added to the local graph. If comparing multiple samples, the graphs are augmented with all new alleles from all samples at the same time.

\section{Optimal VCF reference construction for multi-genome comparison}

In the compare step of pandora (see Fig. 2D), we enable continuity of downstream analysis by outputting genotype information in the conventional VCF [24]. In this format, each row (record) describes possible alternative allele sequence(s) at a position in a (single) reference genome and information about the type of sequence variant. A column for each sample details the allele seen in that sample, often along with details about the support from the data for each allele.

To output graph variation, we first select a path through the graph to be the reference sequence and describe any variation within the graph with respect to this path as shown in Fig. 3. We use the chromosome field to detail the local graph within the PanRG in which a variant lies, and the position field to give the position in the chosen reference path sequence for that graph. In addition, we output the reference path sequences used as a separate file.

For a collection of samples, we want small differences between samples to be recorded as short alleles in the VCF file rather than longer alleles with shared flanking sequence as shown in Fig. 3B. We therefore choose the reference path for each local graph to be maximally close to the sample mosaic paths. To do this, we make a copy of the $k$-mer graph and increment the coverage along each sample mosaic path, producing 


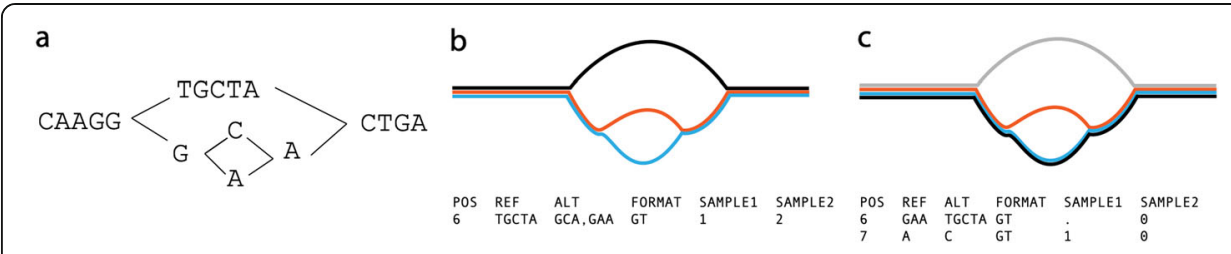

Fig. 3 The representation problem. A A local graph with sequence explicitly shown. B, C The same graph with black reference path and alternate alleles in different colors, and the corresponding VCF records. In $\mathbf{B}$, the black reference path is distinct from both alleles. The blue/red SNP then requires flanking sequence in order to allow it to have a coordinate. The SNP is thus represented as two ALT alleles, each 3 bases long, and the user is forced to notice they only differ in one base. $\mathbf{C}$ The reference follows the blue path, thus enabling a more succinct and natural representation of the SNP

a graph with higher weights on paths shared by more samples. We reuse the mosaic path-finding algorithm (see "Methods") with a modified probability function defined such that the probability of a node is proportional to the number of samples covering it. This produces a dataset-dependent VCF reference able to succinctly describe segregating variation in the cohort of genomes under analysis.

\section{Constructing a PanRG of E. coli}

We chose to evaluate pandora on the recombining bacterial species, E. coli, whose pangenome has been heavily studied [7, 29-32]. MSAs for gene clusters curated with panX [33] from 350 RefSeq assemblies were downloaded from http://pangenome.de on 3 May 2018. MSAs for intergenic region clusters based on 228 E. coli ST131 genome sequences were previously generated with Piggy [34] for their publication. While this panel of intergenic sequences does not reflect the full diversity within $E$. coli, we included them as an initial starting point. This resulted in an E. coli PanRG containing local graphs for 23,051 genes and 14,374 intergenic regions. Pandora took $15 \mathrm{~m}$ in CPU time (11 m in runtime with 16 threads) and 12.9 GB of RAM to index the PanRG. As one would expect from the U-shaped gene frequency distribution, many of the genes were rare in the $578(=350+228)$ input genomes, and so $59 \% / 44 \%$ of the genic/ intergenic graphs were linear, with just a single allele.

\section{Constructing an evaluation set of diverse genomes}

We first demonstrate that using a PanRG reduces hard bias when comparing a diverse set of $20 \mathrm{E}$. coli samples by comparison with standard single-reference variant callers. We selected samples from across the phylogeny (including phylogroups A, B2, D and F [35]) where we were able to obtain both long- and short-read sequence data from the same isolate.

We used Illumina-polished long-read assemblies as truth data, masking positions where the Illumina data did not support the assembly (see "Methods"). As comparators, we used SAMtools [36] (the "classical" variant caller based on pileups) and Freebayes [37] (a haplotype-based caller which reduces soft reference bias, wrapped by snippy [38]) for Illumina data, and medaka [39] and nanopolish [40] for Nanopore data. In all cases, we ran the reference-based callers with 24 carefully selected reference genomes (see "Methods" and Fig. 4). We defined a "truth set" of 618,305 segregating variants by performing all pairwise whole genome alignments of the 20 truth assemblies, collecting 


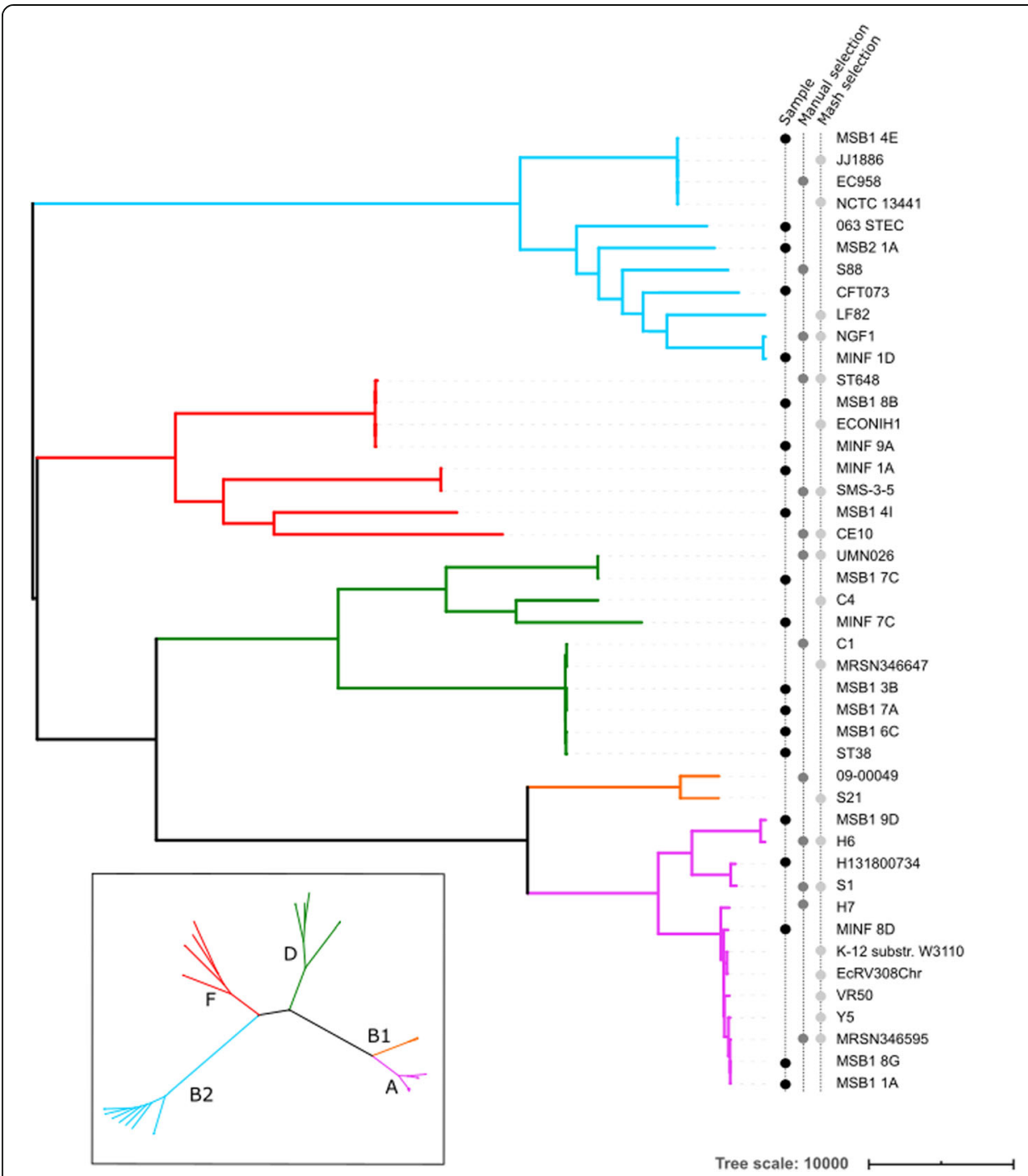

Fig. 4 Phylogeny of 20 diverse $E$. coli along with references used for benchmarking single-reference variant callers. The $20 \mathrm{E}$. coli under study are labelled as samples in the left-hand of three vertical label-lines. Phylogroups (clades) are labelled by color of branch, with the key in the inset. References were selected from RefSeq as being the closest to one of the 20 samples as measured by Mash, or manually selected from a tree (see "Methods"). Two assemblies from phylogroup B1 are in the set of references, despite there being no sample in that phylogroup

SNP variants between the pairs, and deduplicating them by clustering into equivalence classes. Each class, or pan-variant, represents the same variant found at different coordinates in different genomes (see "Methods"). We evaluated error rate (proportion of VCF records which are incorrect, see "Methods"), pan-variant recall (PVR, proportion of segregating sites in the truth set discovered) and average allelic recall (AvgAR, average of the proportion of alleles of each pan-variant that are found). To clarify the definitions, consider a toy example. Suppose we have three genes, each with one SNP between them. The first gene is rare, present in $2 / 20$ genomes. The second gene is at an intermediate frequency, in 10/20 genomes. The third is a strict core gene, present in all genomes. The SNP in the first gene has alleles A,C at 50\% frequency (1 A and $1 \mathrm{C}$ ). The SNP in the second gene has alleles G,T at 50\% frequency ( $5 \mathrm{G}$ and $5 \mathrm{~T}$ ). The SNP 
in the third gene has alleles A,T with $15 \mathrm{~A}$ and $5 \mathrm{~T}$. Suppose a variant caller found the SNP in the first gene, detecting the two correct alleles. For the second gene's SNP, it detected only one $\mathrm{G}$ and one $\mathrm{T}$, failing to detect either allele in the other 8 genomes. For the third gene's SNP, it detected all the 5 T's, but no A. Here, the pan-variant recall would be: $(1+1+0) / 3=0.66-$ i.e., score a 1 if both alleles are found, irrespective of how often- and the average allelic recall would be $(2 / 2+2 / 10+5 / 20) / 3=0.48$. Thus PVR and AvgAR are pan-genome equivalents of standard site discovery power and genotyping accuracy.

\section{Pandora detects rare variation inaccessible to single-reference methods}

First, we evaluate the primary aim of pandora-the ability to access genetic variation within the accessory genome. Figure 5 shows the PVR of SNPs in the truth set broken down by the number of samples the SNP (either allele) is present in. Results are shown for pandora, medaka, and nanopolish using Nanopore sequence data, and Additional file 1: Supplementary Figure 1 shows an almost identical result for pandora, snippy, and SAMtools using Illumina sequence data.

If we restrict our attention to rare variants (present only in 2-5 genomes), we find pandora recovers at least 17.5/24.5/11.6/20.8 k more SNPs than SAMtools/snippy/medaka/nanopolish respectively. As a proportion of rare SNPs in the truth set, this is a lift in PVR of 10.9/15.3/7.2/13.0\% respectively. If, instead of pan-variant recall, we look at the variation of AvgAR across the locus frequency spectrum (see Additional file 1: Supplementary Figure 2), the gap between pandora and the other tools on rare loci is even larger. These observations confirm and quantify the extent to which we are able to recover accessory genetic variation that is inaccessible to single-reference-based methods.

\section{Benchmarking recall, error rate, and dependence on reference}

We show in Fig. 6A,B the Illumina and Nanopore AvgAR/error rate plots for pandora and four single-reference tools with no filters applied. For all of these, we modify only the minimum genotype confidence to move up and down the curves (see "Methods").

We highlight three observations. Firstly, pandora achieves essentially the same recall and error rate for the Illumina and Nanopore data (85\% AvgAR and $0.2-0.3 \%$ error rate

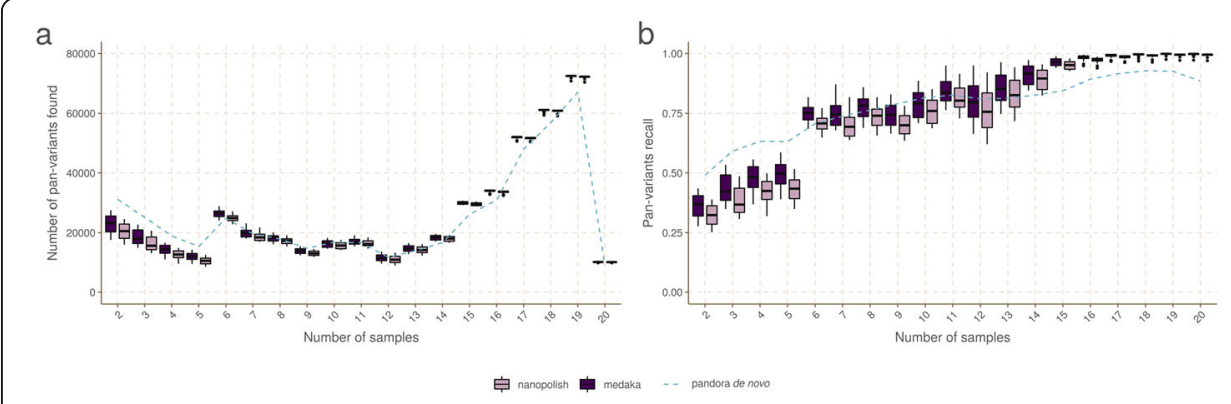

Fig. 5 Pan-variant recall across the locus frequency spectrum. Every SNP occurs in a locus, which is present in some subset of the full set of 20 genomes. SNPs in the golden truth set are broken down by the number of samples the locus is present in. In panel $\mathbf{A}$, we show the absolute count of pan-variants found and in panel $\mathbf{B}$ we show the proportion of pan-variants found (PVR) for pandora (dotted line), nanopolish, and medaka with Nanopore data 


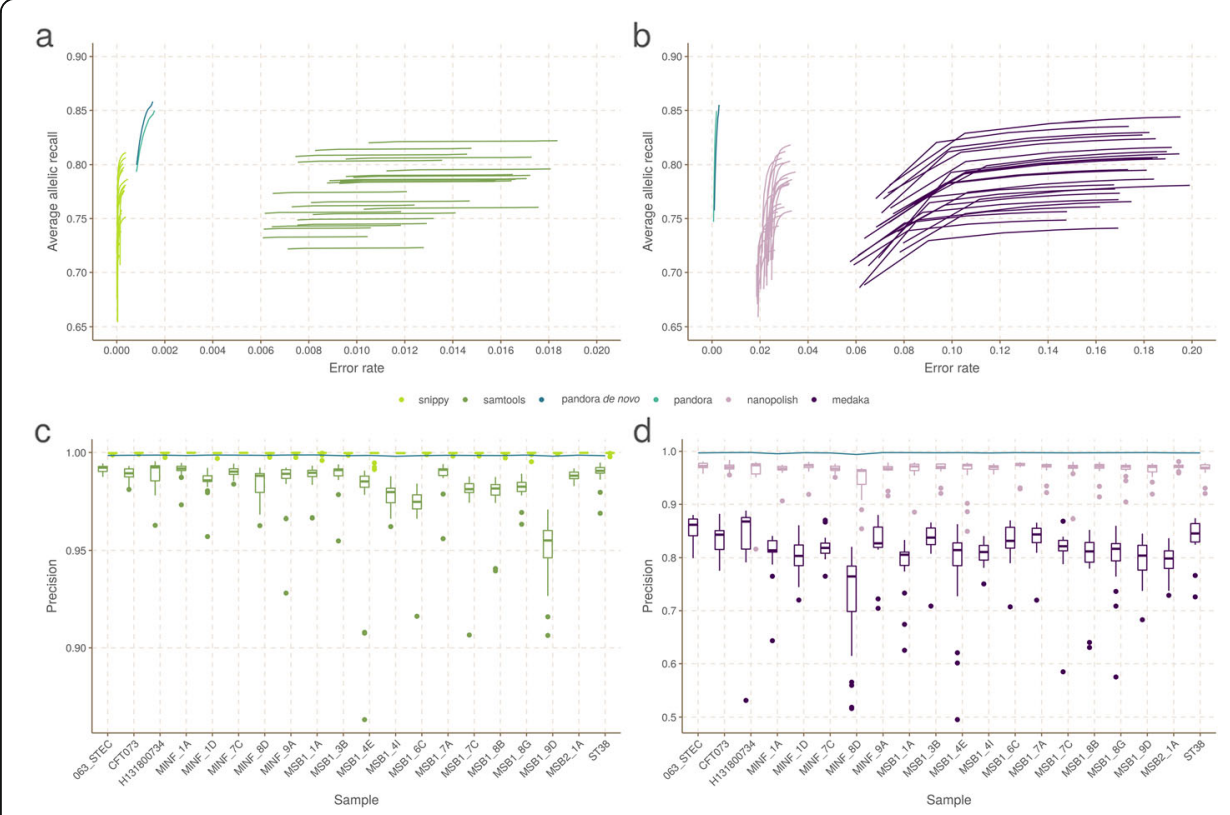

Fig. 6 Benchmarks of recall/error rate and dependence of precision on reference genome, for pandora and other tools on 20-way dataset. A The average allelic recall and error rate curve for pandora, SAMtools, and snippy on 100x of Illumina data. Snippy/SAMtools both run 24 times with the different reference genomes shown in Fig. 4, resulting in multiple lines for each tool (one for each reference). B The average allelic recall and error rate curve for pandora, medaka, and nanopolish on 100x of Nanopore data; multiple lines for medaka/nanopolish, one for each reference genome. Note panels $\mathbf{A}$ and $\mathbf{B}$ have the same $y$-axis scale and limits, but different $x$ axes. C The precision of pandora, SAMtools, and snippy on 100x of Illumina data. The boxplots show the distribution of SAMtools' and snippy's precision depending on which of the 24 references was used, and the blue line connects pandora's results. D The precision of pandora (line plot), medaka, and nanopolish (both boxplots) on 100x of Nanopore data. Note different $y$-axis scale/limits in panels $\mathbf{C}$ and $\mathbf{D}$

at the top-right of the curve, completely unfiltered). Second, choice of reference has a significant effect on both AvgAR and error rate for the single-reference callers; the reference which enables the highest recall does not lead to the best error rate. Third, pandora achieves better AvgAR (85\%) than all other tools (all between 73 and 84\%, see Additional file 1: Supplementary Table 1), and a better error rate (0.2-0.3\%) than SAMtools $(1.0 \%)$, nanopolish (2.4\%), and medaka (14.8\%). However, snippy achieves a significantly better error rate than all other tools $(0.01 \%)$. We confirmed that adding further filters slightly improved error rates, but did not change the overall picture (Additional file 1: Supplementary Figure 3, "Methods", Additional file 1: Supplementary Table 1). The results are also in broad agreement if the PVR is plotted instead of AvgAR (Additional file 1: Supplementary Figure 4). However, these AvgAR and PVR figures are hard to interpret because pandora and the reference-based tools have recall that varies differently across the locus frequency spectrum, as described above. We explore this further below.

We ascribe the similarity between the Nanopore and Illumina performance of pandora to three reasons. First, the PanRG is a strong prior-our first approximation does not contain any Nanopore sequence, but simply uses quasi-mapped reads to find the nearest mosaic in the graph. Second, mapping long Nanopore reads which completely cover entire genes is easier than mapping Illumina data and allows us to filter out erroneous $k$-mers within reads after deciding when a gene is present. Third, this performance is 
only achieved when we use methylation-aware basecalling of Nanopore reads, presumably removing most systematic bias (see Additional file 1: Supplementary Figure 5).

In Fig. 6C,D, we show for Illumina and Nanopore data, the impact of reference choice on the precision of calls on each of the 20 samples. While precision is consistent across all samples for pandora, we see a dramatic effect of reference choice on precision of SAMtools, medaka, and nanopolish. The effect is also detectable for snippy, but to a much lesser extent.

Finally, we measured the performance of locus presence detection, restricting to genes/intergenic regions in the PanRG, so that in principle perfect recall would be possible (see "Methods"). In Additional file 1: Supplementary Figure 6, we show the distribution of locus presence calls by pandora, split by length of locus for Illumina and Nanopore data. Overall, 93.7\%/94.3\% of loci were correctly classified as present or absent for Illumina/Nanopore respectively. Misclassifications were concentrated on small loci (below $500 \mathrm{bp}$ ). While 59.5\%/57.4\% of all loci in the PanRG are small, 75.5\%/75.7\% of false positive calls and 99.1\%/98.5\% of false negative calls are small loci (see Additional file 1: Supplementary Figure 6).

\section{Pandora has consistent results across $E$. coli phylogroups}

We measure the impact of reference bias (and population structure) by quantifying how recall varies in phylogroups $\mathrm{A}, \mathrm{B} 2, \mathrm{D}$, and $\mathrm{F}$ depending on whether the reference genome comes from the same phylogroup.

We plot the results for snippy with 5 exemplar references in Fig. 7A (results for all tools and for all references are in Additional file 1: Supplementary Figures 7-10), showing that single references give 5-10\% higher recall for samples in their own phylogroup than other phylogroups. By comparison, pandora's recall is much more consistent, staying stable at $\sim 90 \%$ for all samples regardless of phylogroup. References in phylogroups A and B2 achieve higher recall in their own phylogroup, but consistently worse than pandora for samples in the other phylogroups (in which the reference does not lie).

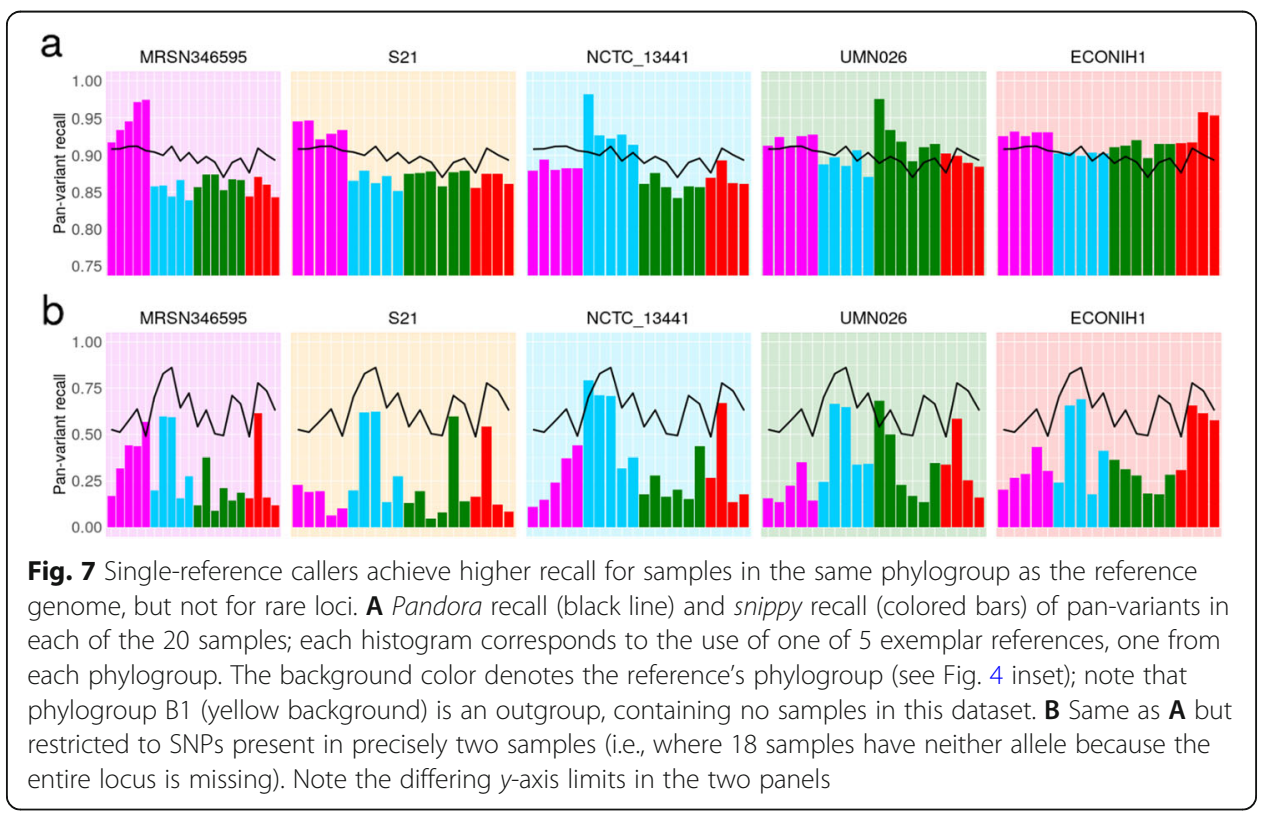


References in the external phylogroup B1, for which we had no samples in our dataset, achieve higher recall for samples in the nearby phylogroup A (see inset, Fig. 4), but lower than pandora for all others. We also see that choosing a reference genome from phylogroup F, which sits intermediate to the other phylogroups, provides the most uniform recall across other groups-2-5\% higher than pandora.

These results will, however, be dominated by the shared, core genome. If we replot Fig. 7A, restricting to variants in loci present in precisely 2 genomes (abbreviated to 2-variants; Fig. 7B), we find that pandora achieves 49-86\% recall for each sample (complete data in Additional file 1: Supplementary Figure 11). By contrast, for any choice of reference genome, the results for single-reference callers vary dramatically per sample, ranging from 4 to $83 \%$ for snippy for example. Most sample-reference pairs (388/480) have recall under 49\% (the lower bound for pandora recall), and there is no pattern of improved recall for samples in the same phylogroup as the reference. Following up that last observation, if we look at which pairs of genomes share 2-variants (Fig. 8), we find there is no enrichment within phylogroups at all. This simply confirms in our data that presence of rare loci is not correlated with the overall phylogeny. For completeness, Additional file 1: Supplementary Animation 2 shows the pandora and snippy recall for all 24 references, split by variant frequency.

\section{Pandora VCF reference is closer to samples than any single reference}

The relationship between phylogenetic distance and gene repertoire similarity is not linear. In fact, 2 genomes in different phylogroups may have more similar accessory genes than 2 in the same phylogroup-as illustrated in the previous section (also see Fig. 3 in Rocha [3]). As a result, it is unclear a priori how to choose a good reference

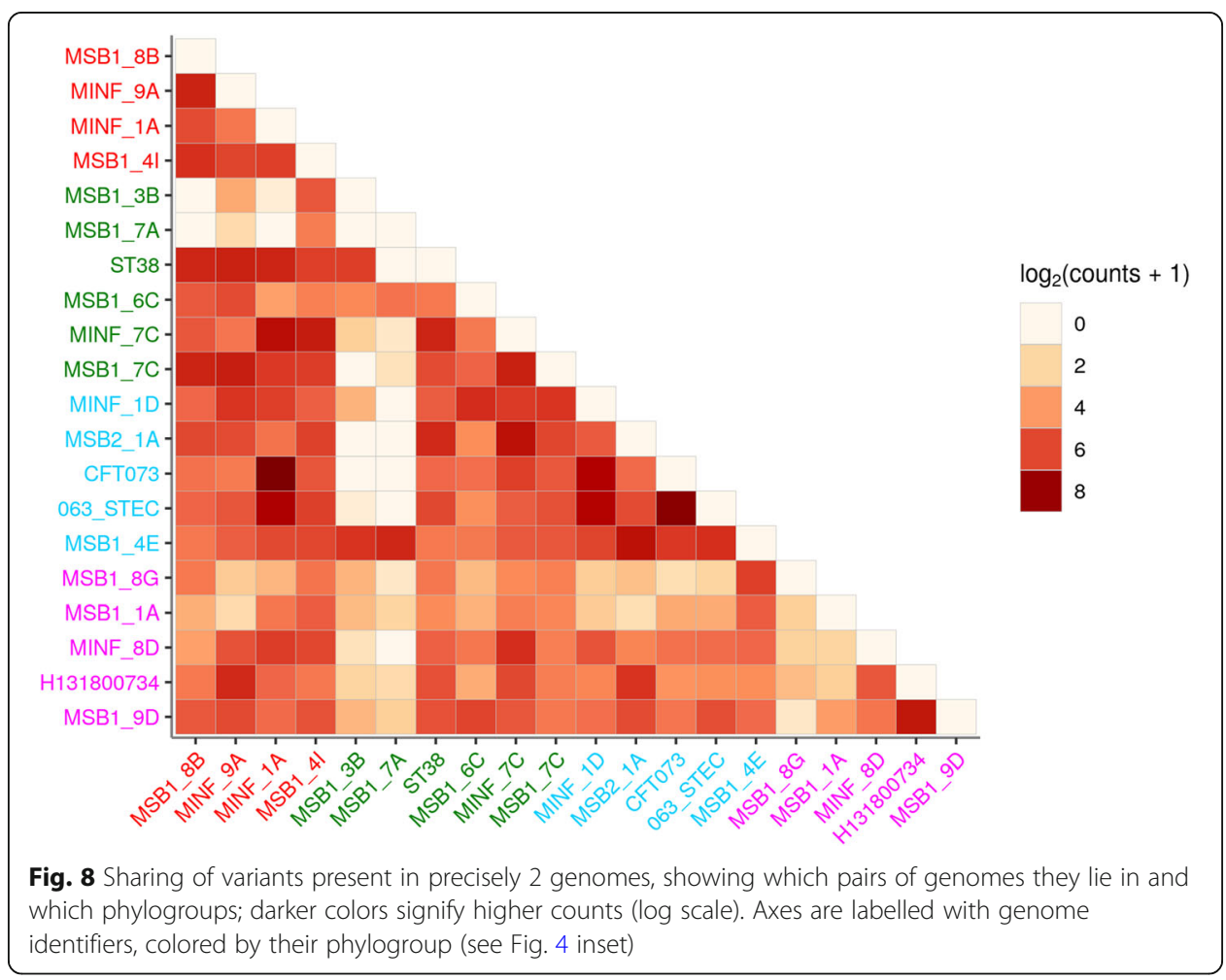


genome for comparison of accessory loci between samples. Pandora specifically aims to construct an appropriate reference for maximum clarity in VCF representation. We evaluate how well pandora is able to find a VCF reference close to the samples under study as follows. We first identified the location of all loci in all the 20 sample assemblies and the 24 references (see "Methods").

We then measured the edit distance between each locus in each of the references and the corresponding version in the 20 samples. We found that the pandora's VCF reference lies within $1 \%$ edit distance (scaled by locus length) of the sample far more than any of the references for loci present in $\leq 9$ samples (Fig. 9; note the log scale). Additional file 1: Supplementary Figure 12 shows a similar result for $0 \%$ edit distance (exact match). In both cases, the improvement is much reduced in the core genome; essentially, in the core, a phylogenetically close reference provides a good approximation, but it is hard to choose a single reference that provides a close approximation to all rare loci. By contrast, pandora is able to leverage its reference panel, and the dataset under study, to find a good approximation.

\section{Computational performance}

We report here the CPU time and maximum RAM consumed by the evaluated tools. All of the single-reference tools analyzed isolates independently, whereas pandora has a subsequent joint analysis step to compare them all; we therefore compare the end-toend performance of pandora analyzing all 20 samples against the mean performance of each single-reference tool (summing all 20 samples, and then averaging over the different reference genomes). In short, pandora took 9.2 CPU hours to analyze the 20

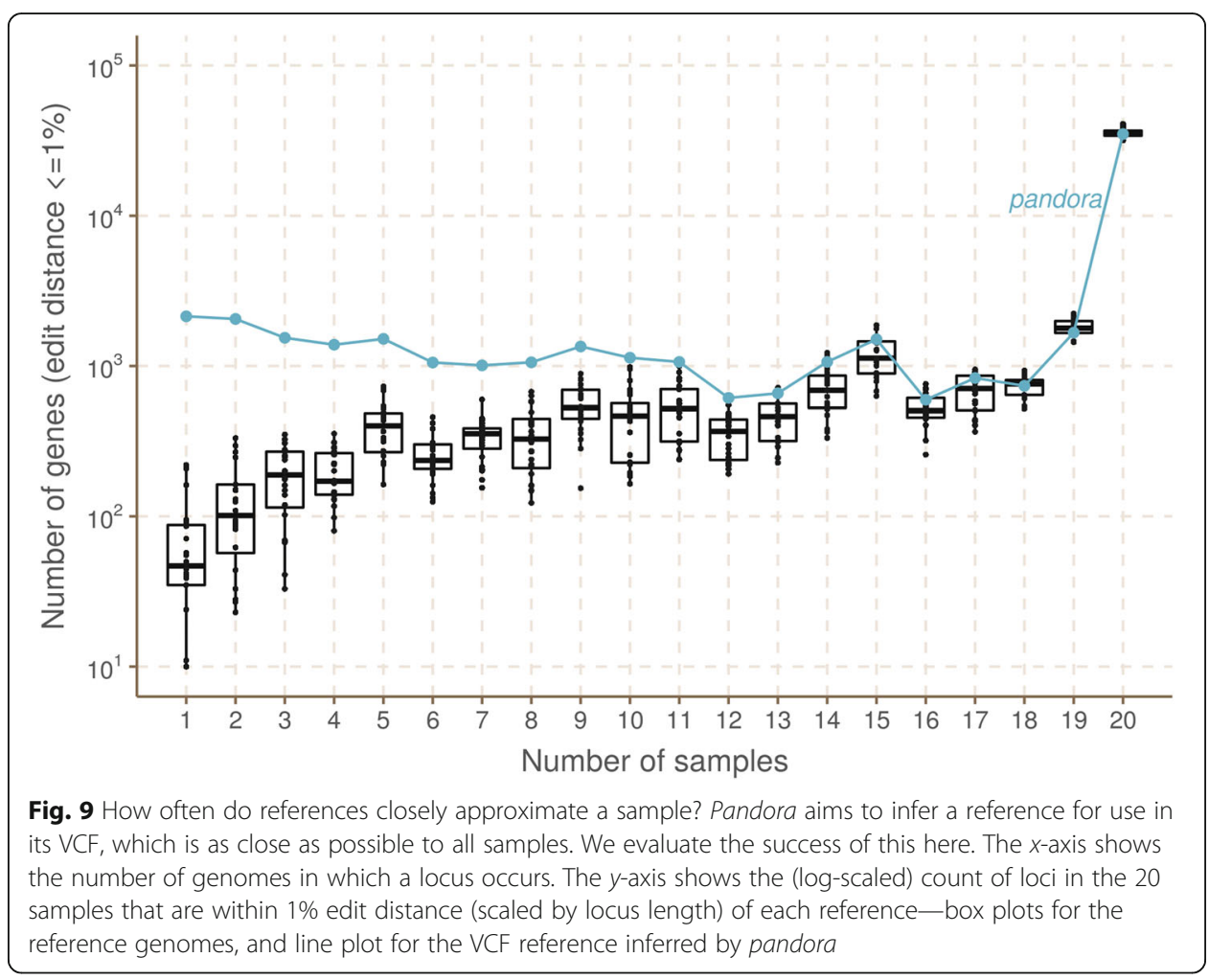


isolates with Illumina data while snippy and SAMtools both took 0.4 CPU hours. With Nanopore data, pandora took 16.4 CPU hours, which is slower than medaka (0.7 CPU hours), but faster than nanopolish (84 CPU hours). In terms of memory usage, for the Illumina data, pandora used a maximum of 13.4 GB of RAM, compared with snippy (3.2 GB), and SAMtools (1.0 GB), whereas for the Nanopore data, pandora used a maximum of $15.7 \mathrm{~GB}$ of RAM, compared with medaka (5.9 GB) and nanopolish (10.4 GB).

\section{Discussion}

Bacteria are the most diverse and abundant cellular life form [41]. Some species are exquisitely tuned to a particular niche (e.g., obligate pathogens of a single host) while others are able to live in a wide range of environments (e.g., E. coli can live on plants, in the earth, or commensally in the gut of various hosts). Broadly speaking, a wider range of environments correlates with a larger pan-genome, and some parts of the gene repertoire are associated with specific niches [42]. Our perception of a pan-genome therefore depends on our sampling of the unknown underlying population structure, and similarly the effectiveness of a PanRG will depend on the choice of reference panel from which it is built.

Many examples from different species have shown that bacteria are able to leverage this genomic flexibility, adapting to circumstance sometimes by using or losing novel genes acquired horizontally, and at other times by mutation. There are many situations where precise nucleotide-level variants matter in interpreting pan-genomes. Some examples include compensatory mutations in the chromosome reducing the fitness burden of new plasmids [43-45]; lineage-specific accessory genes with SNP mutations which distinguish carriage from infection [46]; SNPs within accessory drug resistance genes leading to significant differences in antibiograms [47]; and changes in CRISPR spacer arrays showing immediate response to infection [48, 49]. However, up until now, there has been no automated way of studying non-core gene SNPs at all; still less a way of integrating them with gene presence/absence information. Pandora solves these problems, allowing detection and genotyping of core and accessory variants. It also addresses the problem of what reference to use as a coordinate system, inferring a mosaic "VCF reference" which is as close as possible to all samples under study. We find this gives more consistent SNP calling than any single reference in our diverse dataset. We focussed primarily on Nanopore data when designing pandora and show it is possible to achieve higher quality SNP calling with this data than with current Nanopore tools. The impact of this approach does depend on the dataset under study. We find that, if analyzing closely related samples, then single-reference methods provide improved recall compared with pandora. However, if analyzing more diverse datasets, hard reference bias is a bigger issue for single-reference tools, and pandora offers improved recall.

Prior graph genome work, focussing on soft reference bias (in humans), has evaluated different approaches for selecting alleles for addition to a population graph, based on frequency, avoiding creating new repeats, and avoiding exponential blow-up of haplotypes in clusters of variants [50]. This approach makes sense when you have unphased diploid VCF files and are considering all recombinants of clustered SNPs as possible. However, this is effectively saying we consider the recombination rate to be high enough that all recombinants are possible. Our approach, building from local MSAs 
and only collapsing haplotypes when they agree for a fixed number of bases, preserves more haplotype structure and avoids combinatorial explosion. Another alternative approach was recently taken by Norri et al. [51], inferring a set of pseudo founder genomes from which to build the graph.

With pandora, we break the genome into atomic units which may reorder freely between samples, but within which the degree of variation is more constrained. This approach is directly motivated by our knowledge of the mechanisms underlying genomic flexibility in bacteria. The breadth of diversity we see in these bacteria arises primarily as a result of horizontally acquired DNA which is incorporated into the genome by different forms of recombination. Homologous recombination between closely related sequences may result in allele conversion and in some species contributes as many nucleotide changes as point mutation [52]. As a result, locally we expect sequences to look like mosaics of each other, possibly with additional novel mutations. Genes are acquired (and lost) as a result of homologous or site-specific recombination and at hotspots [53,54]. The dynamics of this are organized [30], and result in global genome mosaicism. The choice of atomic unit used to build each local graph should again be motivated by this underlying biology. A locus should be large enough for its presence and sequence to be useful independently of other graphs, but small enough as to be typically inherited as an entire unit. Biologically speaking, genes fulfil this requirement and there already exist a plethora of tools designed to extract and align genes (and intergenic regions) in a set of bacterial genomes (prokka [55], panaroo [56], roary [57], panX [33], piggy [34]). Operons or groups of genes which co-occur contiguously might also make a good choice, although isolating a set of reference sequences for these regions would be more of a challenge.

Another issue is how to select the reference panel of genomes in order to minimize hard reference bias. One cannot escape the $\mathrm{U}$-shaped frequency distribution; whatever reference panel is chosen, future genomes under study will contain rare genes not present in the PanRG. Given the known strong population structure in bacteria, and the association of accessory repertoires with lifestyle and environment, we would advocate sampling by phylogeny, geography, host species (if appropriate), lifestyle (e.g., pathogenic versus commensal), and/or environment. In this study, we built our PanRG from a biased dataset (RefSeq) which does not attempt to achieve balance across phylogeny or ecology, limiting our pan-variant recall to $49 \%$ for rare variants (see Fig. 5B, Additional file 1: Supplementary Figure 1C). A larger, carefully curated input panel, such as that from Horesh et al. [58], would provide a better foundation and potentially improve results.

A natural question is then to ask if the PanRG should continually grow, absorbing all variants ever encountered. From our perspective, the answer is no-a PanRG with variants at all non-lethal positions would be potentially intractable. The goal is not to have every possible allele in the PanRG-no more than a dictionary is required to contain absolutely every word that has ever been said in a language. As with dictionaries, there is a trade-off between completeness and utility, and in the case of bacteria, the language is far richer than English. The perfect PanRG contains the vast majority of the genes and intergenic regions you are likely to meet, and just enough breadth of allelic diversity to ensure reads map without too many mismatches. Missing alleles should be discoverable by local assembly and added to the graph, allowing multi-sample comparison 
of the cohort under study. This allows one to keep the main PanRG lightweight enough for rapid and easy use.

For bacterial genomes, genotype calls are often used to perform phylogenetic analyses. By detecting accessory variation, three things become possible. First, pragmatically, one can choose clusters of similar genomes based on the cohort-wide core genome, and then by restricting the pandora VCF to genes present in each cluster, reanalyze based on the cluster-specific core genome. Normally this would require choosing a cluster-specific reference, remapping reads and re-running a variant caller, but with pandora all of the necessary data is provided in one step. Secondly, the accessory SNPs provide an extra level of resolution when comparing samples which are very close on a core genome tree, which may be useful. Finally, one cannot represent all pangenome variation in a phylogeny as the evolutionary history is fundamentally not compatible with a simple vertical-inheritance model. However, the pandora output would make ideal material on which to build and test population genetic models.

We finish with potential applications of pandora. First, the PanRG should provide a more interpretable substrate for pan-genome-wide genome-wide association studies, as current methods are forced to either ignore the accessory genome or reduce it to $k$-mers or unitigs [59-61] abstracted from their wider context. Second, it would allow investigation of selection and adaptation of accessory SNPs. Third, if performing prospective surveillance of microbial isolates taken in a hospital, the PanRG provides a consistent and unchanging reference, which will cope with the diversity of strains seen without requiring the user to keep switching reference genome. Finally, if studying a fixed dataset very carefully, then one may not want to use a population PanRG, as it necessarily will miss some rare accessory genes in the dataset. In these circumstances, one could construct a reference graph purely of the genes/intergenic regions present in this dataset.

There are a number of limitations to this study. Firstly, although pandora achieves a gain of recall in rare variation compared with single-reference tools (at least $12-25 \mathrm{k}$ more SNPs in loci present in 2-5 genomes out of 20 depending on choice of tool and reference-a lift of at least $7-15 \%$ in recall), this is offset by $11 \%$ loss of recall at core SNPs. However, the gain in recall of rare variants will increase both with dataset size (due to the U-shaped gene frequency curve) and with a PanRG constructed from either a better-sampled input reference panel, or the dataset itself. By contrast, there is no a priori reason why pandora should miss core SNPs, and this issue will need to be addressed in future work. Finally, by working in terms of atomic loci instead of a monolithic genome-wide graph, pandora opens up graph-based approaches to structurally diverse species (and eases parallelisation) but at the cost of losing genome-wide ordering. At present, ordering can be resolved by (manually) mapping pandora-discovered genes onto whole genome assemblies. However the design of pandora also allows for gene-ordering inference: when Nanopore reads cover multiple genes, the linkage between them is stored in a secondary de Bruijn graph where the alphabet consists of gene identifiers. This results in a huge alphabet, but the $k$-mers are almost always unique, dramatically simplifying "assembly" compared with normal DNA de Bruijn graphs. This work is still in progress and the subject of a future study. In the meantime, pandora provides new ways to access previously hidden variation. 


\section{Conclusions}

The algorithms implemented in pandora provide a solution to the problem of analyzing core and accessory genetic variation across a set of bacterial genomes. This study demonstrates as good SNP genotype error rates with Nanopore as with Illumina data and improved recall of accessory variants. It also shows the benefit of an inferred VCF reference genome over simply picking from RefSeq. The main limitations were the use of a biased reference panel (RefSeq) for building the PanRG, and a slightly lower recall for core SNPs than single-reference toolsboth of which are addressable, not fundamental limitations. This work opens the door to improved analyses of many existing and future bacterial genomic datasets.

\section{Methods}

\section{Local graph construction}

We construct each local graph in the PanRG from an MSA using an iterative partitioning process. The resulting sequence graph contains nested bubbles representing alternative alleles.

Let $A$ be an MSA of length $n$. For each row of the MSA $a=\left\{a_{0}, \ldots, a_{n-1}\right\} \in A$ let $a_{i, j}=$ $\left\{a_{i}, \ldots, a_{j-1}\right\}$ be the subsequence of $a$ in interval $[i, j)$. Let $s(a)$ be the DNA sequence obtained by removing all non-AGCT symbols. We can partition alignment $A$ either vertically by partitioning the interval $[0, n)$ or horizontally by partitioning the set of rows of $A$. In both cases, the partition induces a number of sub-alignments.

For vertical partitions, we define $\operatorname{slice}_{A}(i, j)=\left\{a_{i, j}: a \in A\right\}$. We say that interval $[i, j)$ is a match interval if $j-i \geq m$, where $m=7$ is the default minimum match length, and there is a single non-trivial sequence in the slice, i.e.,

$$
\mid\left\{s(a): a \in \operatorname{slice}_{A}(i, j) \text { and } s(a) \neq \neq^{\prime \prime}\right\} \mid=1 .
$$

Otherwise, we call it a non-match interval.

For horizontal partitions, we use a reference-based approach combined with $K$-means clustering [62] to divide sequences into increasing numbers of clusters $K=2,3, \ldots$ until each cluster meets a "one-reference-like" criterion or until $K=10$. More formally, let $U$ be the set of all $m$-mers (substrings of length $m$, the minimum match length) in $\{s(a)$ : $a \in A\}$. For $a \in A$, we transform sequence $s(a)$ into a count vector $\overline{x_{a}}=\left\{x_{a}{ }^{1}, \ldots, x_{a}|U|\right\}$ where $x_{a}{ }^{i}$ is the count of unique $m$-mer $i \in U$. The $K$-means algorithm partitions $\{s(a)$ : $a \in A\}$ into $K$ clusters $\bar{C}=\left\{C_{1}, \ldots, C_{K}\right\}$ by minimizing the inertia, defined as

$$
\arg \min _{C} \sum_{j=1}^{K} \sum_{\bar{x}_{a} \in C_{j}}\left|\overline{x_{a}}-\mu_{j}\right|^{2}
$$

where $\mu_{j}=\frac{1}{\left|C_{j}\right|} \sum_{\overline{x_{a}} \in C_{j}} \overline{x_{a}}$ is the mean of cluster $j$.

Given a (sub-)alignment $A$, we define the reference of $A, \operatorname{ref}(A)$, to be the concatenation of the most frequent nucleotide at each position of $A$. We say that a $K$-partition is one-reference-like if for the corresponding sub-alignments $A_{1}, \ldots, A_{K}$ the hamming distance between each sequence and its sub-alignment reference

$$
\left|s(a)-r e f\left(A_{i}\right)\right|<d * \operatorname{len}(A) \quad \forall a \in A_{i}
$$

where | | denotes the Hamming distance, and $d$ denotes a maximum hamming distance threshold, set at 0.2 by default. In this case, we accept the partition; otherwise, we look for a $K+1$-partition. 
The recursive algorithm first partitions an MSA vertically into match and non-match intervals. Match intervals are collapsed down to the single sequence they represent. Independently for each non-match interval, the alignment slice is partitioned horizontally into clusters. The same process is then applied to each induced sub-alignment until a maximum number of recursion levels, $r=5$, has been reached. For any remaining alignments, a node is added to the local graph for each unique sequence. See Additional file 1: Supplementary Animation 1 to see an example of this algorithm. We name this algorithm Recursive Cluster and Collapse (RCC). When building the local graph from a MSA, we record and serialize the recursion tree of the RCC algorithm, as well as memoizing all the data in each recursion node. We shall call this algorithm memoized RCC (MRCC). Once the MRCC recursion tree is generated, we can obtain the local graph through a pre-order traversal of the tree (which is equivalent to the call order of the recursive functions in an execution of the RCC algorithm). To update the local graph with new alleles using the MRCC algorithm, we can deserialize the recursion tree from disk, infer in which leaves of the recursion tree the new alleles should be added, add the new alleles in bulk, and then update each modified leaf. This leaf update operation consists of updating just the subaligment of the leaf (which is generally a small fraction of the whole MSA) with the new alleles using MAFFT [63] and recomputing the recursion at the leaf node. All of this is implemented in the make_prg repository (see "Code availability").

\section{$(w, k)$-minimizers of graphs}

We define $(w, k)$-minimizers of strings as in Li [27]. Let $\phi: \Sigma^{k} \rightarrow \mathfrak{R}$ be a $k$-mer hash function and let $\pi: \Sigma^{*} \times\{0,1\} \rightarrow \Sigma^{*}$ be defined such that $\pi(s, 0)=s$ and $\pi(s, 1)=\bar{s}$, where $\bar{s}$ is the reverse complement of $s$. Consider any integers $k \geq w>0$. For window start position $0 \leq j \leq|s|-w-k+1$, let

$$
T_{j}=\left\{\pi\left(s_{p, p+k}, r\right): j \leq p<j+w, r \in\{0,1\}\right\}
$$

be the set of forward and reverse-complement $k$-mers of $s$ in this window. We define a $(w, k)$-minimizer to be any triple $(h, p, r)$ such that

$$
h=\phi\left(\pi\left(s_{p, p+k}, r\right)\right)=\min \left\{\phi(t): t \in T_{j}\right\} .
$$

The set $W(s)$ of $(w, k)$-minimizers for $s$, is the union of minimizers over such windows:

$$
W(s)=\bigcup_{0 \leq j \leq|s|-w-k+1}\left\{(h, p, r): h=\min \left\{\phi(t): t \in T_{j}\right\}\right\} .
$$

We extend this definition intuitively to an acyclic sequence graph $G=(V, E)$. Define $|v|$ to be the length of the sequence associated with node $v \in V$ and let $i=(v, a, b), 0 \leq$ $a \leq b \leq|v|$ represent the sequence interval $[a, b)$ on $v$. We define a path in $G$ by

$$
\bar{p}=\left\{\left(i_{1}, \ldots, i_{m}\right):\left(v_{j}, v_{j+1}\right) \in E \text { and } b_{j} \equiv\left|v_{j}\right| \text { for } 1 \leq j<m\right\} .
$$

This matches the intuitive definition for a path in a sequence graph except that we allow the path to overlap only part of the sequence associated with the first and last nodes. We will use $s_{\bar{p}}$ to refer to the sequence along the path $\bar{p}$ in the graph. 
Let $\bar{q}$ be a path of length $w+k-1$ in $G$. The string $s \bar{q}$ contains $w$ consecutive $k$-mers for which we can find the $(w, k)$-minimizer(s) as before. We therefore define the $(w, k)$ minimizer(s) of the graph $G$ to be the union of minimizers over all paths of length $w+$ $k-1$ in $G$ :

$$
W(G)=\bigcup_{\bar{q} \in G:|\bar{q}|=w+k-1}\left\{(h, \bar{p}, r): h=\min \left\{\phi(t): t \in T_{\bar{q}}\right\}\right\} .
$$

\section{Local graph indexing with $(w, k)$-minimizers}

To find minimizers for a graph, we use a streaming algorithm as described in Additional file 1: Supplementary Algorithm 1. For each minimizer found, it simply finds the next minimizer(s) until the end of the graph has been reached.

Let $\operatorname{walk}(v, i, w, k)$ be a function which returns all vectors of $w$ consecutive $k$-mers in $G$ starting at position $i$ on node $v$. Suppose we have a vector of $k$-mers $x$. Let $\operatorname{shift}(x)$ be the function which returns all possible vectors of $k$-mers which extend $x$ by one $k$-mer. It does this by considering possible ways to walk one letter in $G$ from the end of the final $k$-mer of $x$. For a vector of $k$-mers of length $w$, the function minimize $(x)$ returns the minimizing $k$-mers of $x$.

We define $K$ to be a $k$-mer graph with nodes corresponding to minimizers $(h, \bar{p}, r)$. We add edge $(u, v)$ to $K$ if there exists a path in $G$ for which $u$ and $v$ are both minimizers and $v$ is the first minimizer after $u$ along the path. Let $K \leftarrow a d d(s, t)$ denote the addition of nodes $s$ and $t$ to $K$ and the directed edge $(s, t)$. Let $K \leftarrow a d d(s, T)$ denote the addition of nodes $s$ and $t \in T$ to $\mathrm{K}$ as well as directed edges $(s, t)$ for $t \in T$, and define $K \leftarrow \operatorname{add}(S, t)$ similarly.

The resulting PanRG index stores a map from each minimizing $k$-mer hash value to the positions in all local graphs where that $(w, k)$-minimizer occurred. In addition, we store the induced $k$-mer graph for each local graph.

\section{Quasi-mapping reads}

We infer the presence of PanRG loci in reads by quasi-mapping. For each read, a sketch of $(w, k)$-minimizers is made, and these are queried in the index. For every $(w, k)$ minimizer shared between the read and a local graph in the PanRG index, we define a hit to be the coordinates of the minimizer in the read and local graph and whether it was found in the same or reverse orientation. We define clusters of hits from the same read, local graph, and orientation if consecutive read coordinates are within a certain distance. If this cluster is of sufficient size, the locus is deemed to be present and we keep the hits for further analysis. Otherwise, they are discarded as noise. The default for this "sufficient size" is at least 10 hits and at least 1/5th the length of the shortest path through the $k$-mer graph (Nanopore) or the number of $k$-mers in a read sketch (Illumina). Note that there is no requirement for all these hits to lie on a single path through the local graph. A further filtering step is therefore applied after the sequence at a locus is inferred to remove false positive loci, as indicated by low mean or median coverage along the inferred sequence by comparison with the global average coverage. This quasi-mapping procedure is described in pseudocode in Additional file 1: Supplementary Algorithm 2. 
Initial sequence approximation as a mosaic of references

For each locus identified as present in the set of reads, quasi-mapping provides (filtered) coverage information for nodes of the directed acyclic $k$-mer graph. We use these to approximate the sequence as a mosaic of references as follows. We model $k$ mer coverage with a negative binomial distribution and use the simplifying assumption that $k$-mers are read independently. Let $\Theta$ be the set of possible paths through the $k$ mer graph, which could correspond to the true genomic sequence from which reads were generated. Let $r+s$ be the number of times the underlying DNA was read by the machine, generating a $k$-mer coverage of $s$, and $r$ instances where the $k$-mer was sequenced with errors. Let $1-p$ be the probability that a given $k$-mer was sequenced correctly. For any path $\theta \in \Theta$, let $\left\{X_{1}, \ldots, X_{M}\right\}$ be independent and identically distributed random variables with probability distribution $f\left(x_{i}, r, p\right)=\frac{\Gamma(r+s)}{\Gamma(r) s !} p^{r}(1-p)^{s}$, representing

the $k$-mer coverages along this path. Since the mean and variance are $\frac{(1-p) r}{p}$ and $\frac{(1-p) r}{p^{2}}$, we solve for $r$ and $p$ using the observed $k$-mer coverage mean and variance across all $k$ mers in all graphs for the sample. Let $D$ be the $k$-mer coverage data seen in the read dataset. We maximize the score $\hat{\theta}=\{\arg \max l(\theta \mid D)\}_{\theta \in \Theta}$ where $l(\theta \mid D)=\frac{1}{M} \sum_{i=1}^{M}$ $\log f\left(s_{i}, r, p\right)$, where $s_{i}$ is the observed coverage of the $i$-th $k$-mer in $\theta$. This score is an approximation to a log likelihood, but averages over (up to) a fixed number of $k$-mers in order to retain sensitivity over longer paths in our $\mathrm{C}++$ implementation. By construction, the $k$-mer graph is directed and acyclic so this maximization problem can be solved with a dynamic programming algorithm (for pseudocode, see Additional file 1: Supplementary Algorithm 3).

For choices of $w \leq k$ there is a unique sequence along the discovered path through the $k$-mer graph (except in rare cases within the first or last $w-1$ bases). We use this closest mosaic of reference sequences as an initial approximation of the sample sequence.

\section{De novo variant discovery}

The first step in our implementation of local de novo variant discovery in genome graphs is finding the candidate regions of the graph that show evidence of dissimilarity from the sample's reads.

\section{Finding candidate regions}

The input required for finding candidate regions is a local graph, $n$, within the PanRG, the maximum likelihood path of both sequence and $k$-mers in $n, l m p_{n}$ and $k m p_{n}$ respectively, and a padding size $w$ for the number of positions surrounding the candidate region to retrieve.

We define a candidate region, $r$, as an interval within $n$ where coverage on $l m p_{n}$ is less than a given threshold, $c$, for more than $l$ and less than $m$ consecutive positions. $m$ acts to restrict the size of variants we are able to detect. If set too large, the following steps become much slower due to the combinatorial expansion of possible paths. For a given read, $s$, that has a mapping to $r$ we define $s_{r}$ to be the subsequence of $s$ that maps to $r$, including an extra $w$ positions either side of the mapping. We define the pileup $P_{r}$ as the set of all $s_{r} \in r$. 


\section{Enumerating paths through candidate regions}

For $r \in R$, where $R$ is the set of all candidate regions, we construct a de Bruijn graph $G_{r}$ from the pileup $P_{r}$ using the GATB library [64]. $A_{L}$ and $A_{R}$ are defined as sets of $k$-mers to the left and right of $r$ in the local graph. They are anchors to allow re-insertion of new sequences found by de novo discovery into the local graph. If we cannot find an anchor on both sides, then we abandon de novo discovery for $r$. We use sets of $k$-mers for $A_{L}$ and $A_{R}$, rather than a single anchor $k$-mer, to provide redundancy in the case where sequencing errors cause the absence of some $k$-mers in $G_{r}$. Once $G_{r}$ is built, we define the start anchor $k$-mer, $a_{L}$, as the first $k$-mer in $A_{L}$ that is also in $G_{r}$. Likewise, we define the end anchor $k$-mer, $a_{R}$, as the first $k$-mer in $A_{R}$ that is also in $G_{r}$.

$T_{r}$ is the spanning tree obtained by performing depth-first search (DFS) on $G_{n}$, beginning from node $a_{L}$. We define $p_{r}$ as a path, from the root node $a_{L}$ of $T_{r}$ and ending at node $a_{R}$, which fulfils the two conditions: (1) $p_{r}$ is shorter than the maximum allowed path length; (2) no more than $k$ nodes along $p_{r}$ have coverage $<f e_{r}$ where $e_{r}$ is the expected $k$-mer coverage for $r$ and $f$ is $n_{r} * S$, where $n_{r}$ is the number of iterations of path enumeration for $r$ and $s$ is a step size ( 0.1 by default).

$V_{r}$ is the set of all $p_{r}$. If $\left|V_{r}\right|$ is greater than a predefined threshold, then we have too many candidate paths, and we decide to filter more aggressively: $f$ is incremented by $s-$ effectively requiring more coverage for each $p_{r}$-and $V_{r}$ is repopulated. If $f>1.0$, then de novo discovery is abandoned for $r$.

\section{Pruning the path-space in a candidate region}

As we operate on both accurate and error-prone sequencing reads, the number of valid paths in $G_{r}$ can be very large. Primarily, this is due to cycles that can occur in $G_{r}$ and exploring paths that will never reach our required end anchor $a_{R}$. In order to reduce the path-space within $G_{r}$, we prune paths based on multiple criteria. Critically, this pruning happens at each step of the graph walk (path-building).

We used a distance-based optimisation based on Rizzi et al. [65]. In addition to $T_{r}$, obtained by performing DFS on $G_{r}$, we produce a distance map $D_{r}$ that results from running reversed breadth-first search (BFS) on $G_{r}$, beginning from node $a_{R}$. We say reversed $\mathrm{BFS}$ as we explore the predecessors of each node, rather than the successors. $D_{r}$ is implemented as a binary search tree where each node in the tree represents a $k$-mer in $G_{r}$ that is reachable from $a_{R}$ via reversed BFS. Each node additionally has an integer attached to it that describes the distance from that node to $a_{R}$.

We can use $D_{r}$ to prune the path-space by (1) for each node $n \in p_{r}$, we require $n \in D_{r}$ and (2) requiring $a_{R}$ be reached from $n$ in, at most, $i$ nodes, where $i$ is defined as the maximum allowed path length minus the number of nodes walked to reach $n$.

If one of these conditions is not met, we abandon $p_{r}$. The advantage of this pruning process is that we never explore paths that will not reach our required endpoint within the maximum allowed path length and when caught in a cycle, we abandon the path once we have made too many iterations around the cycle.

\section{Graph-based genotyping and optimal reference construction for multi-genome comparison}

We use graph-based genotyping to output a comparison of samples in a VCF. A path through the graph is selected to be the reference sequence, and graph variation is 
described with respect to this reference. The chromosome field then details the local graph and the position field gives the position within the chosen reference sequence for possible variant alleles. The reference path for each local graph is chosen to be maximally close to the set of sample mosaic paths. This is achieved by reusing the mosaic path-finding algorithm detailed in Additional file 1: Supplementary Algorithm 3 on a copy of the $k$-mer graph with coverages incremented along each sample mosaic path, and a modified probability function defined such that the probability of a node is proportional to the number of samples covering it. This results in an optimal path, which is used as the VCF reference for the multi-sample VCF file.

For each sample and site in the VCF file, the mean forward and reverse coverage on $k$-mers tiling alleles is calculated. A likelihood is then independently calculated for each allele based on a Poisson model. An allele $A$ in a site is called if: (1) $A$ is on the sample mosaic path (i.e., it is on the maximum likelihood path for that sample); (2) $A$ is the most likely allele to be called based on the previous Poisson model. Every allele not in the sample mosaic path will not satisfy (1) and will thus not be called. In the uncommon event where an allele satisfies (1), but not (2), we have an incompatibility between the global and the local choices, and then the site is genotyped as null.

\section{Comparison of variant callers on a diverse set of E. coli}

\section{Sample selection}

We used a set of 20 diverse E. coli samples for which matched Nanopore and Illumina data and a high-quality assembly were available. These are distributed across 4 major phylogroups of $E$. coli as shown in Fig. 4. Of these, 16 were isolated from clinical infections and rectal screening swabs in ICU patients in an Australian hospital [66]. One is the reference strain CFT073 that was resequenced and assembled by the REHAB consortium [67]. One is from an ST216 cardiac ward outbreak (identifier: H131800734); the Illumina data was previously obtained [68], and we did the Nanopore sequencing (see below). The two final samples were obtained from Public Health England: one is a Shiga toxin encoding E. coli (we used the identifier O63) [69], and the other an enteroaggregative E. coli (we used the identifier ST38) [70]. Coverage data for these samples can be found in Additional file 1: Supplementary Table 2.

\section{PanRG construction}

MSAs for gene clusters curated with panX [33] from 350 RefSeq assemblies were downloaded from http://pangenome.de on 3 May 2018. MSAs for intergenic region clusters based on 228 E. coli ST131 genome sequences were previously generated with Piggy [34] for their publication. The PanRG was built using make_prg. Three loci (GC00000027_2, GC00004221 and GC00000895_r1_r1_1) out of 37,428 were excluded because pandora did not complete in reasonable time $(\sim 24 \mathrm{~h})$ once de novo variants were added.

\section{Nanopore sequencing of sample $H 131800734$}

DNA was extracted using a Blood \& Cell Culture DNA Midi Kit (Qiagen, Germany) and prepared for Nanopore sequencing using kits EXP-NBD103 and SQK-LSK108. 
Sequencing was performed on a MinION Mk1 Shield device using a FLO-MIN106 R9.4 Spoton flowcell and MinKNOW version 1.7.3, for $48 \mathrm{~h}$.

\section{Nanopore basecalling}

Recent improvements to the accuracy of Nanopore reads have been largely driven by improvements in basecalling algorithms [71]. For comparison, 4 samples were basecalled with the default (methylation unaware) model and with the methylation-aware, high-accuracy model provided with the proprietary guppy basecaller (version 3.4.5). Additional file 1: Supplementary Figure 5 shows the effect of methylation-aware Nanopore basecalling on the AvgAR/error rate curve for pandora with/without novel variant discovery via local assembly for those 4 samples. With normal basecalling, local de novo assembly increases the error rate substantially from 0.22 to $0.60 \%$, with a negligible increase in recall, from 89.1 to $90.1 \%$, whereas with methylation-aware basecalling it increases the recall from 89.5 to $90.6 \%$ and just slightly increases the error rate from 0.18 to $0.22 \%$. On the basis of this, demultiplexing of the subsequent basecalled data was performed using the same methylation-aware version of the guppy software suite with barcode kits EXP-NBD104 and EXP-NBD114 and an option to trim the barcodes from the output.

\section{Phylogenetic tree construction}

Chromosomes were aligned using MAFFT [63] v7.467 as implemented in Parsnp [72] v1.5.3. Gubbins v2.4.1 was used to filter for recombination (default settings), and phylogenetic construction was carried out using RAxML [73] v8.2.12 (GTR + GAMMA substitution model, as implemented in Gubbins [74]).

\section{Reference selection for mapping-based callers}

A set of references was chosen for testing single-reference variant callers using two standard approaches, as follows. First, a phylogeny was built containing our 20 samples and 243 reference genomes from RefSeq. Then, for each of our 20 samples, the nearest RefSeq E. coli reference was found using Mash [26]. Second, for each of the 20 samples, the nearest RefSeq reference in the phylogeny was manually selected; sometimes one RefSeq assembly was the closest to more than one of the 20. At an earlier stage of the project, there had been another sample (making a total of 21) in phylogroup B1; this was discarded when it failed quality filters (data not shown). Despite this, the Mash/ manual selected reference genomes were left in the set of mapping references, to evaluate the impact of mapping to a reference in a different phylogroup to all 20 of our samples.

\section{Construction of truth assemblies}

16/20 samples were obtained with matched Illumina and Nanopore data and a hybrid assembly. Sample H131800734 was assembled using the hybrid assembler Unicycler [75] with PacBio and Illumina reads followed by polishing with the PacBio reads using Racon [76], and finally with Illumina reads using Pilon [77]. A small $1 \mathrm{~kb}$ artefactual contig was removed from the H131800734 assembly due to low quality and coverage. 
In all cases, we mapped the Illumina data to the assembly and masked all positions where the pileup of Illumina reads did not support the assembly.

\section{Construction of a comprehensive and filtered truth set of pairwise SNPs}

All pairwise comparisons of the 20 truth assemblies were performed with varifier (https://github.com/iqbal-lab-org/varifier), using subcommand make_truth_vcf. In summary, varifier compares two given genomes (referenced as G1 and G2) twice-first using dnadiff [78] and then using minimap2/paftools [28]. The two output sets of pairwise SNPs are then joined and filtered. We create one sequence probe for each allele (a sequence composed of the allele and 50 bases of flank on either side taken from G1) and then map both to $\mathrm{G} 2$ using minimap2. We then evaluate these mappings to verify if the variant found is indeed correct (TP) or not (FP) as follows. If the mapping quality is zero, the variant is discarded to avoid paralogs/duplicates/repeats that are inherently hard to assess. We then check for mismatches in the allele after mapping and confirm that the called allele is the better match.

\section{Constructing a set of ground truth pan-genome variants}

When seeking to construct a truth set of all variants within a set of bacterial genomes, there is no universal coordinate system. We start by taking all pairs of genomes and finding the variants between them, and then need to deduplicate them-e.g., when a variant between genomes 1 and 2 is the same as a variant between genomes 3 and 4 , they should be identified; we define "the same" in terms of genome, coordinate and allele. An allele $A$ in a position $P_{A}$ of a chromosome $C_{A}$ in a genome $G_{A}$ is defined as a triple $A=\left(G_{A}, C_{A}, P_{A}\right)$. A pairwise variant $P w V=\left\{A_{1}, A_{2}\right\}$ is defined as a pair of alleles that describes a variant between two genomes, and a pan-genome variant $\operatorname{PgV}=\left\{A_{1}\right.$, $\left.A_{2}, \ldots, A_{n}\right\}$ is defined as a set of two or more alleles that describes the same variant between two or more genomes. A pan-genome variant $\operatorname{PgV}$ can also be defined as a set of pairwise variants $\operatorname{PgV}=\left\{P w V_{1}, P w V_{2}, \ldots, P w V_{n}\right\}$, as we can infer the set of alleles of $\operatorname{PgV}$ from the pairs of alleles in all these pairwise variants. Note that pan-genome variants are thus able to represent rare and core variants. Given a set of pairwise variants, we seek a set of pan-genome variants satisfying the following properties:

1. [Surjection]:

a. Each pairwise variant is in exactly one pan-genome variant;

b. A pan-genome variant contains at least one pairwise variant;

2. [Transitivity]: if two pairwise variants $P w V_{1}$ and $P w V_{2}$ share an allele, then $P w V_{1}$ and $P w V_{2}$ are in the same pan-genome variant $P g V$.

We model the above problem as a graph problem. We represent each pairwise variant as a node in an undirected graph $G$. There is an edge between two nodes $n_{1}$ and $n_{2}$ if $n_{1}$ and $n_{2}$ share an allele. Each component (maximal connected subgraph) of $G$ then defines a pan-genome variant, built from the set of pairwise variants in the component, satisfying all the properties previously described. Therefore, the set of components of $G$ defines the set of pan-genome variants $P$. However, a pan-genome variant in $P$ could (i) have more than one allele stemming from a single genome, due to a duplication/repeat; 
(ii) represent biallelic, triallelic, or tetrallelic SNPs/indels. For this evaluation, we chose to have a smaller, but more reliable set of pan-genome variants, and thus we filtered $P$ by restricting it to the set of pan-genome variants $P^{\prime}$ defined by the variants $P g V \in P$ such that (i) $\operatorname{PgV}$ has at most one allele stemming from each genome; (ii) $\operatorname{PgV}$ is a biallelic SNP. $P^{\prime}$ is the set of 618,305 ground truth filtered pan-genome variants that we extracted by comparing and deduplicating the pairwise variants present in our 20 samples and that we use to evaluate the recall of all the tools in this paper. Additional file 1: Supplementary Figure 13 shows an example summarizing the described process of building pan-genome variants from a set of pairwise variants.

\section{Subsampling read data and running all tools}

All read data was randomly subsampled to $100 \times$ coverage using rasusa-the pipeline is available at https:/github.com/iqbal-lab-org/subsampler. A snakemake [79] pipeline to run the pandora workflow with and without de novo discovery (see Fig. 2D) is available at https://github.com/iqbal-lab-org/pandora_workflow. A snakemake pipeline to run snippy, SAMtools, nanopolish, and medaka on all pairwise combinations of 20 samples and 24 references is available at https://github.com/iqbal-lab-org/variant_callers_ pipeline.

\section{Evaluating VCF files}

Calculating precision Given a variant/VCF call made by any of the evaluated tools, where the input were reads from a sample (or several samples, in the case of pandora) and a reference sequence (or a PanRG, in the case of pandora), we perform the following steps to assess how correct a call is:

1. Construct a probe for the called allele, consisting of the sequence of the allele flanked by $150 \mathrm{bp}$ on both sides from the reference sequence. This reference sequence is one of the 24 chosen references for snippy, SAMtools, nanopolish, and medaka; or the multi-sample inferred VCF reference for pandora;

2. Map the probe to the sample sequence using BWA-MEM [80];

3. Remove multi-mappings by looking at the Mapping Quality (MAPQ) measure [36] of the SAM records. If the probe is mapped uniquely, then its mapping passes the filter. If there are multiple mappings for the probe, we select the mapping $m_{1}$ with the highest MAPQ if the difference between its MAPQ and the second highest MAPQ exceeds 10. If $m_{1}$ does not exist, then there are at least two good enough mappings, and it is ambiguous to choose which one to evaluate. In this case, we prefer to be conservative and filter this call (and all its related mappings) out of the evaluation;

4. We further remove calls mapping to masked regions of the sample sequence, in order to not evaluate calls lying on potentially misassembled regions;

5. Now we evaluate the mapping, giving the call a continuous precision score between 0 and 1 . We look only at the alignment of the called allele (i.e., we ignore the flanking sequences alignment) and give a score as follows: number of matches / alignment length. 
Finally, we compute the precision for the tool by summing the score of all evaluated calls and dividing by the number of evaluated calls. Note that here we evaluate all types of variants, including SNPs and indels.

Calculating recall We perform the following steps to calculate the recall of a tool:

1. Apply the VCF calls to the associated reference using the VCF consensus builder (https://github.com/leoisl/vcf_consensus_builder), creating a mutated reference with the variants identified by the tool;

2. Build probes for each allele of each pan-genome variant previously computed (see section "Constructing a set of ground truth pan-genome variants");

3. Map all pan-genome variants' probes to the mutated reference using BWA-MEM;

4. Evaluate each probe mapping, which is classified as a TP only if all bases of the allele were correctly mapped to the mutated reference. In the uncommon case where a probe multimaps, it is enough that one of the mappings are classified as TP;

5. Finally, as we now know for each pan-genome variant which of its alleles were found, we calculate both the pan-variant recall and the average allelic recall as per section "Pandora detects rare variation inaccessible to single-reference methods."

Filters Given a VCF file with likelihoods for each genotype, the genotype confidence is defined as the log likelihood of the maximum likelihood genotype minus the log likelihood of the next best genotype. Thus a confidence of zero means the two most likely alleles are equally likely, and high-quality calls have higher confidences. In the recall/ error rate plots of Fig. 6A,B, each point corresponds to the error rate and recall computed as previously described, on a genotype confidence (gt-conf) filtered VCF file with a specific threshold for minimum confidence.

We also show the same plot with further filters applied in Additional file 1: Supplementary Figure 3. The filters were as follows. For Illumina data: for pandora, a minimum coverage filter of $5 \times$, a strand bias filter of 0.05 (minimum $5 \%$ of reads on each strand), and a gaps filter of 0.8 were applied. The gaps filter means at least $20 \%$ of the minimizer $k$-mers on the called allele must have coverage above $10 \%$ of the expected depth. As snippy has its own internal filtering, no filters were applied. For SAMtools, a minimum coverage filter of $5 \times$ was used. For Nanopore data, for pandora, a minimum coverage filter of $10 \times$, a strand bias filter of 0.05 , and a gaps filter of 0.6 were used. For nanopolish, we applied a coverage filter of $10 \times$. We were unable to apply a minimum coverage filter for medaka due to a software bug that prevents annotating the VCF file with coverage information.

\section{Locus presence and distance evaluation}

For all loci detected as present in at least one sample by pandora, we mapped the multi-sample inferred reference to all 20 sample assemblies and 24 reference sequences, to identify their true locations. To be confident of these locations, we employed a strict mapping using bowtie2 [81] and requiring end-to-end alignments. From the mapping of all loci to all samples, we computed a truth locus presence-absence matrix and compared it with pandora's locus presence-absence matrix, classifying each pandora locus 
call as true/false positive/negative. Additional file 1: Supplementary Figure 6 shows these classifications split by locus length. Having the location of all loci in all the 20 sample assemblies and the 24 references, we then computed the edit distance between them.

\section{Supplementary Information}

The online version contains supplementary material available at https://doi.org/10.1186/s13059-021-02473-1.

Additional file 1: A: Supplementary figures 1-13. B: Supplementary tables 1-2. C: Supplementary algorithms 1-3.

D: Detailed data availability. E: Supplementary animations 1-2.

Additional file 2: Review history.

\section{Acknowledgements}

We are grateful to the REHAB consortium (https://modmedmicro.nsms.ox.ac.uk/rehab/) and the Transmission of Carbapenemase-producing Enterobacteriaceae (TRACE) study investigators for sharing sequencing data (for CFT073 and H131800734) in support of this work. We would like to thank Sion Bayliss and Ed Thorpe for discussions and help with Piggy. We are grateful to Kelly Wyres for sharing sequence data for the Australian samples, and to Tim Dallman and David Greig for sharing their data from Public Health England. We would like to thank the following for helpful conversations during the prolonged genesis of this project: Gil McVean, Derrick Crook, Eduardo Rocha, Bill Hanage, Ed Feil, Sion Bayliss, Ed Thorpe, Richard Neher, Camille Marchet, Rayan Chikhi, Kat Holt, Claire Gorrie, Rob Patro, Fatemeh Almodaresi, Nicole Stoesser, Liam Shaw, Phelim Bradley, and Sorina Maciuca. We would also like to thank the reviewers, with whose help the study was significantly improved.

\section{Review history}

The review history is available as additional file 2.

\section{Peer review information}

Andrew Cosgrove was the primary editor of this article and managed its editorial process and peer review in collaboration with the rest of the editorial team.

\section{Authors' contributions}

RMC designed and implemented the fundamental data structures, and RCC, map and compare algorithms. MBH designed and implemented the de novo variant discovery component. LL designed and implemented the update algorithm for RCC. LL optimized the codebase. RMC, MBH, and LL designed and implemented (several iterations of) the evaluation pipeline, one component of which was written by $\mathrm{MH}$. BL reimplemented and improved the RCC codebase. JH, SG, and LP sequenced 18/20 of the samples. LWR, MBH, LL, KM, and ZI analyzed and visualized the 20way data. ZI designed the study. ZI and RMC wrote the bulk of the paper, LL and MBH wrote sections, and all authors read and improved drafts. The author(s) read and approved the final manuscript.

\section{Funding}

RMC was funded by a Wellcome Trust PhD studentship (105279/Z/14/Z), and ZI was partially funded by a Wellcome Trust/Royal Society Sir Henry Dale Fellowship (102541/Z/13/Z). Open Access funding enabled and organized by Projekt DEAL.

\section{Availability of data and materials}

Reproducibility

All input data for our analyses, including panX's and Piggy's MSAs, PanRG, reference sequences, and sample data are publicly available (see "Data availability" below). The pandora code, as well as all code needed to reproduce these analyses are also publicly available (see "Code availability" below). Software environment reproducibility is achieved using Python virtual environments if all dependencies and source code are in Python, and otherwise using Docker [82] containers run with Singularity [83]. We ran pandora version 0.9.1 and make_prg version 0.2.0 in this study. The exact commit of all repositories used to obtain the results in this paper can be retrieved with the git branch or tag pandora_paper_update_31_03_2021.

Data availability

Gene MSAs from panX, and intergenic MSAs from Piggy: [84]

E. coli PanRG: [85]

Accession identifiers or Figshare links for the sample and reference assemblies, and Illumina and Nanopore reads are listed in Section D of Additional file 1.

Input packages containing all data to reproduce all analyses described in "Results" are also available in Section D of Additional file 1.

Code availability

All code is open source and available under an MIT license:

make_prg (RCC graph construction and update algorithm): https://github.com/leoisl/make_prg

pandora: https://github.com/rmcolq/pandora

varifier: https://github.com/iqbal-lab-org/varifier

Pan-genome variations pipeline taking a set of assemblies and returning a set of filtered pan-genome variants:

https://github.com/iqbal-lab-org/pangenome_variations 
pandora workflow: https://github.com/iabal-lab-org/pandora_workflow

Run snippy, SAMtools, nanopolish and medaka pipeline: https://github.com/iqbal-lab-org/variant_callers_pipeline Evaluation pipeline (recall/error rate curves, etc.): https://github.com/iqbal-lab-org/pandora_paper_roc Locus presence and distance from reference pipeline: https://github.com/iqbal-lab-org/pandora_gene_distance A master repository to reproduce everything in this paper, marshalling all of the above: https://github.com/iqbal-laborg/paper_pandora2020_analyses

Although all containers are hosted on https://hub.docker.com/ (for details, see https://github.com/iqbal-lab-org/paper_ pandora2020_analyses/blob/master/scripts/pull_containers/pull_containers.sh), and are downloaded automatically during the pipelines' execution, we also provide Singularity [83] containers (converted from Docker containers) at https://doi.org/10.6084/m9.figshare.14779257.v1.

Frozen packages with all the code repositories for pandora and the analysis framework can be found at [86].

\section{Declarations}

\section{Ethics approval and consent to participate}

Not applicable

\section{Consent for publication}

Not applicable

\section{Competing interests}

The authors declare that they have no competing interests

\section{Author details}

'European Bioinformatics Institute, Hinxton, Cambridge CB10 1SD, UK. 'Wellcome Trust Centre for Human Genetics, University of Oxford, Roosevelt Drive, Oxford, UK. ${ }^{3}$ Institute of Evolutionary Biology, Ashworth Laboratories, University of Edinburgh, Edinburgh, UK. ${ }^{4}$ Nuffield Department of Medicine, University of Oxford, Oxford, UK. ${ }^{5}$ Department of Infectious Diseases, Central Clinical School, Monash University, Melbourne, Victoria 3004, Australia. ${ }^{6}$ Department of Zoology, University of Oxford, Mansfield Road, Oxford, UK.

\section{Received: 26 November 2020 Accepted: 19 August 2021}

Published online: 14 September 2021

\section{References}

1. Lynch M, Ackerman MS, Gout J-F, Long H, Sung W, Thomas WK, et al. Genetic drift, selection and the evolution of the mutation rate. Nat Rev Genet. Nature Publishing Group. 2016;17(11):704-14. https://doi.org/10.1038/nrg.2016.104.

2. Didelot X, Maiden MCJ. Impact of recombination on bacterial evolution. Trends Microbiol. 2010;18(7):315-22. https://doi. org/10.1016/j.tim.2010.04.002.

3. Rocha EPC. Neutral Theory, Microbial practice: challenges in bacterial population genetics. Mol Biol Evol. Oxford Academic. 2018;35(6):1338-47. https://doi.org/10.1093/molbev/msy078.

4. Fraser C, Alm EJ, Polz MF, Spratt BG, Hanage WP. The bacterial species challenge: making sense of genetic and ecological diversity. Science. American Association for the Advancement of Science. 2009;323(5915):741-6. https://doi. org/10.1126/science.1159388.

5. Mira A, Ochman H, Moran NA. Deletional bias and the evolution of bacterial genomes. Trends Genet. Elsevier. 2001; 17(10):589-96. https://doi.org/10.1016/S0168-9525(01)02447-7.

6. Domingo-Sananes MR, Mclnerney JO. Selection-based model of prokaryote pangenomes. bioRxiv. Cold Spring Harbor Laboratory; 2019;782573.

7. Gordienko EN, Kazanov MD, Gelfand MS. Evolution of pan-genomes of Escherichia coli, Shigella spp., and Salmonella enterica. J Bacteriol. American Society for Microbiology Journals. 2013;195:2786-92.

8. Lobkovsky AE, Wolf Yl, Koonin EV. Gene frequency distributions reject a neutral model of genome evolution. Genome Biol Evol. 2013;5(1):233-42. https://doi.org/10.1093/gbe/evt002.

9. Bolotin E, Hershberg R. Gene loss dominates as a source of genetic variation within clonal pathogenic bacterial species. Genome Biol Evol. Oxford Academic. 2015;7(8):2173-87. https://doi.org/10.1093/gbe/evv135.

10. Haegeman B, Weitz JS. A neutral theory of genome evolution and the frequency distribution of genes. BMC Genomics. 2012;13(1):196. https://doi.org/10.1186/1471-2164-13-196.

11. Hadfield J, Croucher NJ, Goater RJ, Abudahab K, Aanensen DM, Harris SR. Phandango: an interactive viewer for bacterial population genomics. Bioinformatics. Oxford Academic. 2018;34(2):292-3. https://doi.org/10.1093/bioinformatics/btx610.

12. Garrison E, Sirén J, Novak AM, Hickey G, Eizenga JM, Dawson ET, et al. Variation graph toolkit improves read mapping by representing genetic variation in the reference. Nat Biotechnol. Nature Publishing Group. 2018;36(9):875-9. https://doi. org/10.1038/nbt.4227.

13. Maciuca S, Elias C Del O, Mcvean G, lqbal Z. A natural encoding of genetic variation in a Burrows-Wheeler transform to enable mapping and genome inference. algorithms in bioinformatics [Internet]. Springer, Cham; 2016 [cited 2020 Dec 9]. p. 222-33. Available from: https://doi.org/10.1007/978-3-319-43681-4_18

14. Eggertsson HP, Jonsson H, Kristmundsdottir S, Hjartarson E, Kehr B, Masson G, et al. Graphtyper enables population-scale genotyping using pangenome graphs. Nat Genet. Nature Publishing Group. 2017;49(11):1654-60. https://doi.org/10.103 8/ng.3964.

15. Eggertsson HP, Kristmundsdottir S, Beyter D, Jonsson H, Skuladottir A, Hardarson MT, et al. GraphTyper2 enables population-scale genotyping of structural variation using pangenome graphs. Nature Communications. Nature Publishing Group; 2019;10:5402.

16. Rautiainen M, Marschall T. GraphAligner: rapid and versatile sequence-to-graph alignment. bioRxiv. Cold Spring Harbor Laboratory; 2019;810812. 
17. Schneeberger K, Hagmann J, Ossowski S, Warthmann N, Gesing S, Kohlbacher O, et al. Simultaneous alignment of short reads against multiple genomes. Genome Biology. 2009;10(9):R98. https://doi.org/10.1186/gb-2009-10-9-r98.

18. Rabbani L, Müller J, Weigel D. An algorithm to build a multi-genome reference. bioRxiv. Cold Spring Harbor Laboratory. 2020;2020(04):11.036871.

19. The Computational Pan-Genomics Consortium. Computational pan-genomics: status, promises and challenges. Briefings Bioinformatics. 2018;19:118-35.

20. Rautiainen M, Marschall T. Aligning sequences to general graphs in $\mathrm{O}(\mathrm{V}+\mathrm{mE})$ time. bioRxiv. Cold Spring Harbor Laboratory; 2017;216127.

21. Sirén J, Monlong J, Chang X, Novak AM, Eizenga JM, Markello C, et al. Genotyping common, large structural variations in 5,202 genomes using pangenomes, the Giraffe mapper, and the vg toolkit. bioRxiv. Cold Spring Harbor Laboratory. 2021;2020(12):04.412486.

22. Chen S, Krusche P, Dolzhenko E, Sherman RM, Petrovski R, Schlesinger F, et al. Paragraph: a graph-based structural variant genotyper for short-read sequence data. Genome Biology. 2019;20(1):291. https://doi.org/10.1186/s13059-019-1909-7.

23. Sibbesen JA, Maretty L, Krogh A. Accurate genotyping across variant classes and lengths using variant graphs. Nat Genet. 2018;50(7):1054-9. https://doi.org/10.1038/s41588-018-0145-5.

24. Danecek P, Auton A, Abecasis G, Albers CA, Banks E, DePristo MA, et al. The variant call format and VCFtools. Bioinformatics. Oxford Academic. 2011;27(15):2156-8. https://doi.org/10.1093/bioinformatics/btr330.

25. Roberts M, Hayes W, Hunt BR, Mount SM, Yorke JA. Reducing storage requirements for biological sequence comparison. Bioinformatics. Oxford Academic. 2004;20(18):3363-9. https://doi.org/10.1093/bioinformatics/bth408.

26. Ondov BD, Treangen TJ, Melsted P, Mallonee AB, Bergman NH, Koren S, et al. Mash: fast genome and metagenome distance estimation using MinHash. Genome Biology. 2016;17(1):132. https://doi.org/10.1186/s13059-016-0997-x.

27. Li H. Minimap and miniasm: fast mapping and de novo assembly for noisy long sequences. Bioinformatics. Oxford Academic. 2016;32(14):2103-10. https://doi.org/10.1093/bioinformatics/btw152.

28. Li H. Minimap2: pairwise alignment for nucleotide sequences. Bioinformatics. 2018;34(18):3094-100. https://doi.org/10.1 093/bioinformatics/bty191.

29. Touchon M, Perrin A, De SJAM, Vangchhia B, Burn S, O'Brien CL, et al. Phylogenetic background and habitat drive the genetic diversification of Escherichia coli. PLOS Genetics. Public Library of Science. 2020;16:e1008866.

30. Touchon M, Hoede C, Tenaillon O, Barbe V, Baeriswyl S, Bidet P, et al. Organised genome dynamics in the Escherichia coli species results in highly diverse adaptive paths. PLOS Genetics. Public Library of Science. 2009;5:e1000344.

31. Decano AG, Downing T. An Escherichia coli ST131 pangenome atlas reveals population structure and evolution across 4,071 isolates. Sci Rep. Nature Publishing Group. 2019;9:17394.

32. Rasko DA, Rosovitz MJ, Myers GSA, Mongodin EF, Fricke WF, Gajer P, et al. The pangenome structure of Escherichia coli: comparative genomic analysis of E. coli commensal and pathogenic isolates. J Bacteriol. American Society for Microbiology Journals. 2008;190:6881-93.

33. Ding W, Baumdicker F, Neher RA. panX: pan-genome analysis and exploration. Nucleic Acids Res. Oxford Academic. 2018;46:e5-5.

34. Thorpe HA, Bayliss SC, Sheppard SK, Feil EJ. Piggy: a rapid, large-scale pan-genome analysis tool for intergenic regions in bacteria. Gigascience [Internet]. Oxford Academic; 2018 [cited 2020 Jul 3];7. Available from: https://academic.oup.com/ gigascience/article/7/4/giy015/4919733

35. Clermont O, Christenson JK, Denamur E, Gordon DM. The Clermont Escherichia coli phylo-typing method revisited: improvement of specificity and detection of new phylo-groups. Environ Microbiol Rep. 2013;5(1):58-65. https://doi.org/1 $0.1111 / 1758-2229.12019$.

36. Li H, Handsaker B, Wysoker A, Fennell T, Ruan J, Homer N, et al. The Sequence Alignment/Map format and SAMtools. Bioinformatics. Oxford Academic. 2009;25(16):2078-9. https://doi.org/10.1093/bioinformatics/btp352.

37. Garrison E, Marth G. Haplotype-based variant detection from short-read sequencing. arXiv:12073907 [q-bio] [Internet]. 2012 [cited 2020 Jul 3]; Available from: http://arxiv.org/abs/1207.3907

38. Snippy [Internet]. Available from: https://github.com/tseemann/snippy

39. Medaka [Internet]. Available from: https://github.com/Nanoporetech/medaka

40. Loman NJ, Quick J, Simpson JT. A complete bacterial genome assembled de novo using only nanopore sequencing data. Nat Methods. Nature Publishing Group. 2015;12(8):733-5. https://doi.org/10.1038/nmeth.3444.

41. Louca S, Mazel F, Doebeli M, Parfrey LW. A census-based estimate of Earth's bacterial and archaeal diversity. PLOS Biology. Public Library of Science. 2019;17:e3000106.

42. Brockhurst MA, Harrison E, Hall JPJ, Richards T, McNally A, MacLean C. The ecology and evolution of pangenomes. Curr Biol. 2019;29(20):R1094-103. https://doi.org/10.1016/j.cub.2019.08.012.

43. Harrison E, Brockhurst MA. Plasmid-mediated horizontal gene transfer is a coevolutionary process. Trends Microbiol. Elsevier. 2012;20(6):262-7. https://doi.org/10.1016/j.tim.2012.04.003.

44. Harrison E, Dytham C, Hall JPJ, Guymer D, Spiers AJ, Paterson S, et al. Rapid compensatory evolution promotes the survival of conjugative plasmids. Mobile Genet Elements. 2016;6(3):e1179074. https://doi.org/10.1080/2159256X.2016.1179074.

45. Loftie-Eaton W, Bashford K, Quinn H, Dong K, Millstein J, Hunter S, et al. Compensatory mutations improve general permissiveness to antibiotic resistance plasmids. Nat Ecol Evol. 2017;1 (9):1354-63. https://doi.org/10.1038/s41559-017-0243-2.

46. Gori A, Harrison OB, Mlia E, Nishihara Y, Chan JM, Msefula J, et al. Pan-GWAS of Streptococcus agalactiae highlights lineage-specific genes associated with virulence and niche adaptation. mBio [Internet]. American Society for Microbiology; 2020 [cited 2020 Jul 16];11. Available from: https://mbio.asm.org/content/11/3/e00728-20

47. Bonnet R. Growing group of extended-spectrum $\beta$-lactamases: the CTX-M enzymes. Antimicrob Agents Chemother. 2004:48(1):1-14. https://doi.org/10.1128/AAC.48.1.1-14.2004.

48. Louwen R, Staals RHJ, Endtz HP, van Baarlen P, van der Oost J. The role of CRISPR-Cas systems in virulence of pathogenic bacteria. Microbiol Mol Biol Rev. American Society for Microbiology. 2014;78(1):74-88. https://doi.org/10.112 8/MMBR.00039-13.

49. Horvath P, Romero DA, Coûté-Monvoisin A-C, Richards M, Deveau H, Moineau S, et al. Diversity, activity, and evolution of CRISPR loci in Streptococcus thermophilus. J Bacteriol. American Society for Microbiology Journals. 2008;190:1401-12. 
50. Pritt J, Chen N-C, Langmead B. FORGe: prioritizing variants for graph genomes. Genome Biology. 2018;19(1):220. https:// doi.org/10.1186/s13059-018-1595-X.

51. Norri T, Cazaux B, Kosolobov D, Mäkinen V. Linear time minimum segmentation enables scalable founder reconstruction. Algorithms for Molecular Biology. 2019;14(1):12. https://doi.org/10.1186/s13015-019-0147-6.

52. Vos M, Didelot X. A comparison of homologous recombination rates in bacteria and archaea. ISME J. 2009;3(2):199-208. https://doi.org/10.1038/ismej.2008.93.

53. Oliveira PH, Touchon M, Cury J, Rocha EPC. The chromosomal organization of horizontal gene transfer in bacteria. Nat Commun. 2017:8(1):841. https://doi.org/10.1038/s41467-017-00808-w.

54. Didelot $X$, Méric $G$, Falush $D$, Darling AE. Impact of homologous and non-homologous recombination in the genomic evolution of Escherichia coli. BMC Genomics. 2012;13(1):256. https://doi.org/10.1186/1471-2164-13-256.

55. Seemann T. Prokka: rapid prokaryotic genome annotation. Bioinformatics. 2014;30(14):2068-9. https://doi.org/10.1093/ bioinformatics/btu153.

56. Tonkin-Hill G, MacAlasdair N, Ruis C, Weimann A, Horesh G, Lees JA, et al. Producing polished prokaryotic pangenomes with the Panaroo pipeline. Genome Biology. 2020;21(1):180. https://doi.org/10.1186/s13059-020-02090-4.

57. Page AJ, Cummins CA, Hunt M, Wong VK, Reuter S, Holden MTG, et al. Roary: rapid large-scale prokaryote pan genome analysis. Bioinformatics. 2015;31(22):3691-3. https://doi.org/10.1093/bioinformatics/btv421.

58. Horesh G, Blackwell G, Tonkin-Hill G, Corander J, Heinz E, Thomson NR. A comprehensive and high-quality collection of E. coli genomes and their genes. bioRxiv. Cold Spring Harbor Laboratory. 2020;2020(09):21.293175.

59. Lees JA, Vehkala M, Välimäki N, Harris SR, Chewapreecha C, Croucher NJ, et al. Sequence element enrichment analysis to determine the genetic basis of bacterial phenotypes. Nat Commun. Nature Publishing Group. 2016;7:1-8.

60. Earle SG, Wu C-H, Charlesworth J, Stoesser N, Gordon NC, Walker TM, et al. Identifying lineage effects when controlling for population structure improves power in bacterial association studies. Nat Microbiol. Nature Publishing Group. 2016;1:1-8.

61. Jaillard M, Lima L, Tournoud M, Mahé P, Van BA, Lacroix V, et al. A fast and agnostic method for bacterial genome-wide association studies: bridging the gap between k-mers and genetic events. PLOS Genetics. Public Library of Science. 2018;14:e1007758.

62. MacQueen J. Some methods for classification and analysis of multivariate observations. The Regents of the University of California; 1967 [cited 2020 Jul 6]. Available from: https://projecteuclid.org/euclid.bsmsp/1200512992

63. Katoh K, Misawa K, Kuma K, Miyata T. MAFFT: a novel method for rapid multiple sequence alignment based on fast Fourier transform. Nucleic Acids Res. 2002;30(14):3059-66. https://doi.org/10.1093/nar/gkf436.

64. Drezen E, Rizk G, Chikhi R, Deltel C, Lemaitre C, Peterlongo P, et al. GATB: Genome Assembly \& Analysis Tool Box. Bioinformatics. Oxford Academic. 2014;30(20):2959-61. https://doi.org/10.1093/bioinformatics/btu406.

65. Rizzi R, Sacomoto G, Sagot M-F. Efficiently listing bounded length st-paths. In: Jan K, Miller M, Froncek D, editors. Combinatorial Algorithms. Cham: Springer International Publishing; 2015. p. 318-29. https://doi.org/10.1007/978-3-319-1 9315-1_28.

66. Wyres KL, Nguyen TNT, Lam MMC, Judd LM, van Vinh CN, Dance DAB, et al. Genomic surveillance for hypervirulence and multi-drug resistance in invasive Klebsiella pneumoniae from South and Southeast Asia. Genome Medicine. 2020; 12(1):11. https://doi.org/10.1186/s13073-019-0706-y.

67. De Maio N, Shaw LP, Hubbard A, George S, Sanderson ND, Swann J, et al. Comparison of long-read sequencing technologies in the hybrid assembly of complex bacterial genomes. Microb Genom. 2019;5.

68. Decraene V, Phan HTT, George R, Wyllie DH, Akinremi O, Aiken Z, et al. A large, refractory nosocomial outbreak of Klebsiella pneumoniae carbapenemase-producing Escherichia coli demonstrates carbapenemase gene outbreaks involving sink sites require novel approaches to infection control. Antimicrob Agents Chemother. 2018;62(12). https:// doi.org/10.1128/AAC.01689-18.

69. Greig D, Dallman T, Jenkins C. Oxford Nanopore sequencing elucidates a novel stx2f carrying prophage in a Shiga toxin producing Escherichia coli(STEC) O63:H6 associated with a case of haemolytic uremic syndrome (HUS). Access Microbiology. Microbiology Society; 2019;1:782.

70. Greig DR, Dallman TJ, Hopkins KL, Jenkins C. MinION nanopore sequencing identifies the position and structure of bacterial antibiotic resistance determinants in a multidrug-resistant strain of enteroaggregative Escherichia coli. Microbial Genomics. Microbiology Society; 2018;4:e000213.

71. Rang FJ, Kloosterman WP, de Ridder J. From squiggle to basepair: computational approaches for improving nanopore sequencing read accuracy. Genome Biology. 2018;19(1):90. https://doi.org/10.1186/s13059-018-1462-9.

72. Treangen TJ, Ondov BD, Koren S, Phillippy AM. The Harvest suite for rapid core-genome alignment and visualization of thousands of intraspecific microbial genomes. Genome Biology. 2014;15(11):524. https://doi.org/10.1186/s13059-014-0524-x.

73. Stamatakis A. RAxML version 8: a tool for phylogenetic analysis and post-analysis of large phylogenies. Bioinformatics. 2014;30(9):1312-3. https://doi.org/10.1093/bioinformatics/btu033.

74. Croucher NJ, Page AJ, Connor TR, Delaney AJ, Keane JA, Bentley SD, et al. Rapid phylogenetic analysis of large samples of recombinant bacterial whole genome sequences using Gubbins. Nucleic Acids Res. 2015;43(3):e15. https://doi.org/1 0.1093/nar/gku1196.

75. Wick RR, Judd LM, Gorrie CL, Holt KE. Unicycler: Resolving bacterial genome assemblies from short and long sequencing reads. PLOS Computational Biology. Public Library of Science; 2017;13:e1005595.

76. Vaser R, Sović I, Nagarajan N, Šikić M. Fast and accurate de novo genome assembly from long uncorrected reads. Genome Res. 2017;27(5):737-46. https://doi.org/10.1101/gr.214270.116.

77. Walker BJ, Abeel T, Shea T, Priest M, Abouelliel A, Sakthikumar S, et al. Pilon: an integrated tool for comprehensive microbial variant detection and genome assembly improvement. PLOS ONE. Public Library of Science. 2014;9:e112963.

78. Kurtz S, Phillippy A, Delcher AL, Smoot M, Shumway M, Antonescu C, et al. Versatile and open software for comparing large genomes. Genome Biol. 2004;5(2):R12. https://doi.org/10.1186/gb-2004-5-2-r12.

79. Köster J, Rahmann S. Snakemake-a scalable bioinformatics workflow engine. Bioinformatics. 2018;34(20):3600. https://doi. org/10.1093/bioinformatics/bty350.

80. Li H. Aligning sequence reads, clone sequences and assembly contigs with BWA-MEM. arXiv:13033997 [q-bio] [Internet]. 2013 [cited 2020 Nov 2]; Available from: http://arxiv.org/abs/1303.3997 
81. Langmead B, Salzberg SL. Fast gapped-read alignment with Bowtie 2. Nat Methods. 2012;9(4):357-9. https://doi.org/10.1 038/nmeth.1923.

82. Merkel D. Docker: lightweight Linux containers for consistent development and deployment. Linux J. 2014;2014:2:2.

83. Kurtzer GM, Sochat V, Bauer MW. Singularity: scientific containers for mobility of compute. PLOS ONE. Public Library of Science; 2017;12:e0177459.

84. Colquhoun R, Hall M, Lima L, Roberts L, Malone K, Hunt M, et al. Pandora: nucleotide-resolution bacterial pan-genomics with reference graphs. Datasets. Gene MSAs. Available from: https://doi.org/10.6084/m9.figshare.14781732.v1

85. Colquhoun R, Hall M, Lima L, Roberts L, Malone K, Hunt M, et al. Pandora: nucleotide-resolution bacterial pan-genomics with reference graphs. Datasets. E coli PanRG. Available from: https://doi.org/10.6084/m9.figshare.14781756.v1

86. Colquhoun R, Hall M, Lima L, Roberts L, Malone K, Hunt M, et al. Pandora: nucleotide-resolution bacterial pan-genomics with reference graphs. Software. Code repositories for pandora and the paper analysis framework. Available from: https://doi.org/10.6084/m9.figshare.14815899.v2

\section{Publisher's Note}

Springer Nature remains neutral with regard to jurisdictional claims in published maps and institutional affiliations.

Ready to submit your research? Choose BMC and benefit from:

- fast, convenient online submission

- thorough peer review by experienced researchers in your field

- rapid publication on acceptance

- support for research data, including large and complex data types

- gold Open Access which fosters wider collaboration and increased citations

- maximum visibility for your research: over $100 \mathrm{M}$ website views per year

At BMC, research is always in progress.

Learn more biomedcentral.com/submissions 